\title{
UN NUEVO ALGORITMO PARA LA OPTIMACIÓN DE ESTRUCTURAS: EL RECOCIDO SIMULADO
}

\author{
(A NEW ALGORITHM FOR THE OPTIMIZATION OF STRUCTURES: SIMULATED \\ ANNEALING)
}

Mariano Vázquez Espí

Fecha de recepción: $13-$ IIII-95

Dpto. de Estructuras de Edificación de la UPM

400-29

de Madrid-España

\begin{abstract}
RESUMEN
La optimación de una función real es un problema recurrente en teoria de estructuras. Si la función es la energía potencial y la optimación se entiende como la búsqueda de su valor minimo, se trata del análisis convencional de estructuras. Si la función es representativa del "coste", su optimación es un método de diseño de la estructura; de hecho,conducirá al "mejor" diseño respecto al "coste" considerado.
\end{abstract}

En tanto que problema matemático general no existe un método de resolución, salvo para ciertos casos particulares en los que la función y sus variables satisfacen condiciones especificas (continuidad, diferenciabilidad, etc.). En numerosos casos de indole práctica no se dan tales condiciones y, en consecuencia, la solución al problema se aproxima, existiendo para ello una variedad de métodos heuristicos.

El recocido simulado es un algoritmo de aproximación a la solución óptima, fundado en una analogía con el comportamiento de sistemas termodinámicos simples y viene siendo utilizado en ciertos problemas de ingeniería. El presente trabajo muestra como usarlo en la teoria de estructuras, señalando sus ventajas (universalidad) asi como sus inconvenientes (lentitud).

Para ello, se describen brevemente los distintos problemas de la teoría de estructuras, asi como aspectos fundamentales de la teoria de algoritmos. Con mayor detalle, se describe la forma matemática del algoritmo de recocido. En ambos casos se emplea un problema paradigmático de optimación: el del viajante de comercio. Finalmente, se muestra el empleo del algoritmo para "aproximar" formas óptimas de cerchas isostáticas.

\begin{abstract}
SUMMARY
The optimization of a real function is a problem that frequently occurs in structural theory. If the function is the potential energy and optimization means search for the minimum, the problem is structural analysis as usual. If the function represents a "cost", its optimization is transformed into a design method for the structure, actually, it can lead to the "better" design with respect to the "cost" considered.

As mathematical problem, no way to solve it is known, but for a few particular cases in which function and variables fulfil specific conditions (continuity, differentiability, etc.). These conditions do not hold at all in practical cases and, consequently, it is possible only to look for approximations, for which several heuristics exist.
\end{abstract}

Simulated annealing is an approximation algorithm towards the optimal solution. The basis is an analogy to the performance of simple themodinamical systems. In fact it is in use for some engineering problems. The present research shows how to use it on structural theory, pointing out its benefits (generality) and its drawbacks (slowness).

With this goal in mind,the different problems of structural theory as well as some aspects of algorithm theory are examined briefly. The mathematical form of the algorithm is shown in more detail. In both cases, the "traveling salesman" problem is used as a paradigm of generic optimization. Finally, simulated annealing is used for approximating the optimal shape of isostatic trusses.

Existe a menudo confusión en cómo debe usarse el análisis en el diseño estructural. El diseñador comprende pronto que la precisión es inútil en algunos casos e importante en otros; y el diseñador experimentado [del mismo modo] comprende claramente que el análisis convencional es una guía pobre para alcanzar proporciones adecuadas...

Hardy Cross, 1936. 
El recocido simulado es un algoritmo en el mismo sentido en que lo son las reglas para multiplicar o dividir dos números, pero se trata de un conjunto de operaciones mucho más complicado, de manera que, en la práctica, sólo puede ejecutarse mediante máquinas de cálculo. Elalgoritmo simula el comportamiento de sistemas físicos simples sometidos a un descenso paulatinoylentodesu temperatura, tal es el caso de la producción de acero laminado o la cristalización de azúcar en soluciones sobresaturadas; tras un lento proceso tales sistemas alcanzan un estado caracterizado por ser mínima su energía, a pesar del comportamiento intrínsecamentealeatorio de sus partículas microscópicas. En ocasiones, tales estados se reconocen por una particular perfección visual; piénsese, por ejemplo, en un macrocristal de azúcar, monoclínico y regular (en teoría). El recocido simulado permite explotar el conocimiento físico de tales procesos con vistas a buscar el mínimo absoluto de cualquier función matemática, que también en ocasiones corresponderáa formas que muestran cualidades apreciables en tanto "ordenadas", "razonables" o "sensatas". El algoritmo no es nuevo y viene siendo empleado con éxito en diversas disciplinas desde hace más de una década.

El propósito principal de este trabajo es ilustrar y divulgar su uso en problemas de la teoría de estructuras. Sin embargo, no se pretende descender a todos los aspectos de detalle, aquéllos que serían necesarios para transformar el algoritmo en un programa concreto de ordenador capaz de resolver un problema particular, detalles que pueden consultarse, por ejemplo, en Press et alii (1988:346-351) o Vázquez (1994).

El plan de la exposición es el siguiente:

1. El recocido simulado es una analogía, de manera que resulta imprescindible mostrar su origen, en primer lugar.

2. El algoritmo permite resolver problemas de optimación, por lo que resulta necesario explorar algunas características peculiares de tales problemas, en particular aquéllas que los convierten en más fáciles o difíciles que otros.

3. A continuación, se explica con detalle la aplicación del algoritmo a un problema de optimación paradigmático.

4. Después, se trazan las líneas maestras de su aplicación a un problema clásico de optimación de estructuras, comentándose algunos de los resultados.

5. Finalmente, se argumenta acerca de la posible utilidad del algoritmo para la teoría de estructuras.

\section{El origen de la analogía}

La palabra recocido denota un método particular para fabricar acero, del tipo empleado para laminar los perfiles utilizados en edificios y máquinas. Sin embargo, recocido se refiere aquí, especialmente, a las características que distinguen este método de fabricación de otros, tales como el temple.

Como es bien sabido, el acero es hierro con pequeñas adiciones de otras sustancias que, alterando su estructura microscópica, le confieren propiedades distintas a las del hierro puro. Dada una cierta composición química del acero, una gran variedad de estructuras o configuraciones de sus átomos son posibles en el estado sólido. Con cierta aproximación, puede decirse que el recocido es el método empleado para obtener la configuración más estable, caracterizada por ser minima su energía interna. Esto es esencialmente cierto, siempre que no se consideren cambios químicos, pues, en otro caso, el acero recocido acaba por oxidarse, adquiriendo un nueva estructura mucho más estable aún.

En esencia, el recocido tiene dos fases. En primer lugar, se eleva la temperatura de la mezcla por encima del punto de fusión, a fin de tornarla líquida, perdiéndose así toda su estructura anterior y aumentando notablemente la movilidad de sus partículas. En segundo lugar, se deja enfriar muy lentamente, a fin de dar oportunidad a que las partículas vuelvan a configurarse de manera ordenada, antes de perder totalmente su movilidad, con el objetivo de que la estructura resultante se aproxime a la forma estable teórica.

Este proceso y sus características son comunes a muchos otros fenómenos, tales como los sucesivos cambios de fase entre un gas y el sólido correspondiente. En particular, al crecimiento de un cristal por precipitación de una sustancia disuelta en agua(tal como la sal oel azúcar). La cristalización puede provocarse bien por un descenso de la temperatura, o bien por evaporación del agua a temperatura constante. Ambos procesos son equivalentes y, si son suficientemente lentos, conducen a un macrocristal en el que, salvo ocasionales defectos, el orden de la celda microscópica básica se reproduce sin interrupción y a larga distancia.

\subsection{Descripción y simulación matemática}

Si no se desea un descripción detallada de los fenómenos anteriores, ecuaciones matemáticas simples y universales dan cuenta de lo esencial de todos ellos, si bien en términos de probabilidad; en la termodinámica clásica juega tal papel la distribución estadística de Maxwell-Boltzmann (Tabla 1). Tales ecuaciones permiten simular dichos fenómenos matemáticamente, sin necesidad de llevar a cabo experimentos reales.

Estas técnicas de simulación tieneñ su origen en las investigaciones de la energía termonūclear, llevadas a cabo desde la Segunda Guerra Mundial en el tristemente 
Tabla 1

\section{Distribución de Maxwell-Boltzmann}

\begin{tabular}{|cl|}
\hline & $\langle\epsilon\rangle=\frac{1}{Z} \sum \epsilon_{i} \exp \left(-\epsilon_{i} / k T\right)$ \\
& $p_{i}=\frac{1}{Z} \exp \left(-\epsilon_{i} / k T\right)$ \\
siendo: $\quad \epsilon_{i}$ & energía de la configuración $i$ \\
$\langle\epsilon\rangle$ & valor medio de la energía \\
$p_{i}$ & probabilidad de la configuración $i$ \\
$T$ & temperatura \\
$k$ & constante de Boltzmann \\
$Z$ & 'suma sobre los estados', constante del sistema en \\
& estudio para cada valor de $T$
\end{tabular}

famoso Laboratorio de Los Alamos, en las que se utilizó la computadora MANIAC, uno de los primitivos modelos con tubos de vacío (HANSON, 1982). Hoy la técnica se conoce como método de Monte Carlo o, mejor aún, como algoritmo de Metropolis, en honor del líder del grupo de investigadores que lousó por vez primera (véase Metrópolis et alii, 1953). Esta segunda denominación es preferible pues se trata de un método de Monte Carlo muy específico. Para la simulación, cada configuración de un sistema físico se describe como un conjunto de variables matemáticas con valores concretos:

$$
\mathrm{X}=\left\{\mathrm{x}_{1}, \mathrm{x}_{2}, \ldots, \mathrm{x}_{\mathrm{g}}, \ldots\right\}
$$

A cada conjunto de valores le corresponde un valor de la energía , que puede calcularse mediante alguna ecuación específica, $\phi=f(\mathrm{X})$, que intenta representar las cualidades esenciales del sistema. Esta ecuación, a su vez, se maneja mediante las ecuaciones generales de la termodinámica, que son independientes del sistema particular, tales como la distribución de Boltzmann citada.

La simulación permite en primer lugar verificar si la representación contenida en la función específica, $f$, es conforme con los datos experimentales disponibles, y si, por tanto, se ha conseguido con ella capturar lo esencial del fenómeno.

Una vez validada la función, puede emplearse también la simulación para predecir el comportamiento del sistema, lo que resulta útil en aquellas condiciones particulares en que la realización de un experimento de laboratorio resultaría difícil, o muy costosa. En particular, podría emplearse la simulación para calcular tanto la configuración más estable o fundamental, como el valor mínimo de la energía correspondiente, lo que se formula habitualmente como:

Encontrar $\mathrm{X}_{0}$ tal que: $\quad \phi\left(\mathrm{X}_{0}\right)=\underset{\mathrm{X}}{\min } \phi(\mathrm{X})$

Este último objetivo es idéntico al de un problema matemático muy general, presente en numerosas disciplinas: la búsqueda del mínimo valor de una función. Debido a ello, durante la década de los ochenta se empleó con éxito la técnica para buscar formas óptimas en una amplia variedad de problemas concretos, tal y como el diseño de circuitos electrónicos, en los que interesa optimar distintas propiedades, como, por ejemplo, la densidad de cableado, la probabilidad de interferencias magnéticas entre componentes o el número de conexiones entre el circuito y su zócalo (Kirkpatrick et alii, 1983).

En consecuencia, el recocido simulado sólo puede considerarse nuevo en lo que respecta a la búsqueda de valores óptimos de estructuras mecánicas, aunque en tanto que algoritmo de propósito general tiene ya una década de antigüedad. Esta novedad debe mirarse con precaución no es imposible que haya sido empleado ya con el propósito de optimar estructuras, pero, de ser así, he sido incapaz de encontrar la referencia pertinente entre el fascinante número de publicaciones científicas que se producen cada año.

\section{Optimación de estructuras}

En la teoría de estructuras hay dos problemas genéricos que preguntan por el mínimo de una función.

Así, por ejemplo, existen innumerables-maneras en que una estructura, tal y como un puente de ferrocarril (Fig. 1), 
puede deformarse, pero, bajo la acción de cargas determinadas, sólo algunas de las deformaciones posibles harán mínima la energía potencial del conjunto de cargas y estructura. Esta clase de problemas, resueltos por el análisis de estructuras, tiene una obvia conexión con los problemas físicos anteriormente citados, por ser la energía la propiedad a la que se presta atención.

Pero, al igual que ocurre con la deformación, muchos diseños de puentes son posibles en cada caso concreto. Desde luego, todos ellos deben ser seguros, no deformarse en exceso, fáciles de mantener y limpiar, etc. La teoría de estructuras no sólo discute el modo de obtener y analizar cualquiera de tales diseños aceptables: desde sus orígenes también pone énfasis en cómo determinar el o los mejores diseños posibles (Fig. 2). La búsqueda del mejor diseño puede convertirse en un problema con igual estructura matemática que el problema de análisis, a condición de que para medir la calidad puede definirse una propiedad con un valor preciso para cada diseño, como el peso de la estructura, por ejemplo.

En ambos casos se trataría de un problema que puede expresarse formalmente con la ecuación (1) y que, por ant pooria ser resuelto con cualquier técnica disponible, por aiejada que esté de nuestro campo concreto de estudio.

\subsection{El problema del viajante}

A fin de destacar algunas características sobresalientes del problema general de optimar una función, puede servir de ilustración un problema paradigmático, conocido como el viajante de comercio, debido, sobre todo, a que puede plantearse muy brevemente:

Dado un conjunto de ciudades, se trata de encontrar el itinerario que permite visitar todas una vez, recorriendo la mínima distancia posible y regresando al final a la ciudad de origen (Fig. 3a).

Sus propiedades sobresalientes son comunes a un numeroso conjunto de problemas y aquí se resaltarán informalmente, aunque su estudio formal se aborda mediante la teoría general de algoritmos y, en particular, mediante la teoria de la NP-compleción (véase, por ejemplo, Garey et Johnson, 1979).

\subsection{Resolubilidad}

Ante cualquier problema la primera cuestión es saber si tiene solución. En el caso del viajante de comercio, puede contestarse afirmativamente.
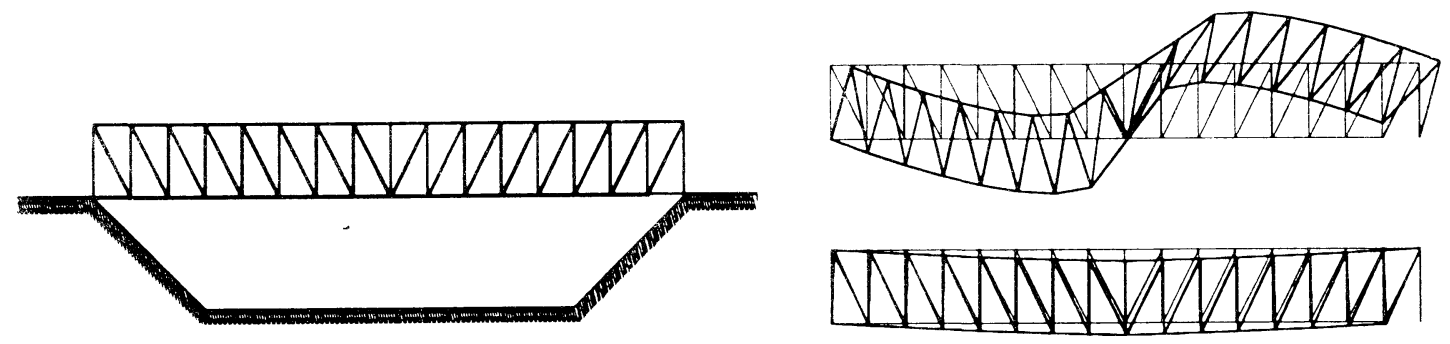

Fig.1.- Análisis de estructuras : A la izquierda, un puente de ferrocarril; a la derecha, dos posibles deformaciones.
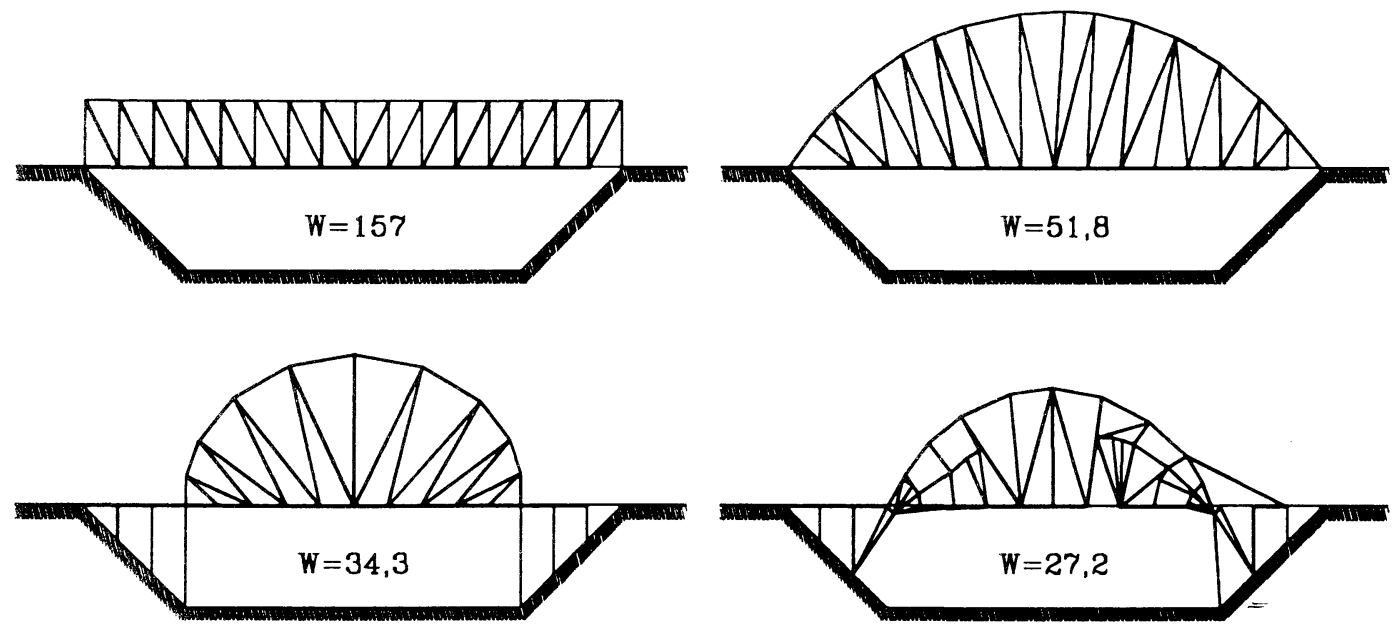

Fig.2.- Diseño de estructuras : Wes proporcional a la cantidad de material necesaria para construir cada diseño. El diseño, abajo y a la derecha, fue encontrado mediante recocido-según se explica más adelante-, partiendo del que figura arriba, a la izquierda. 

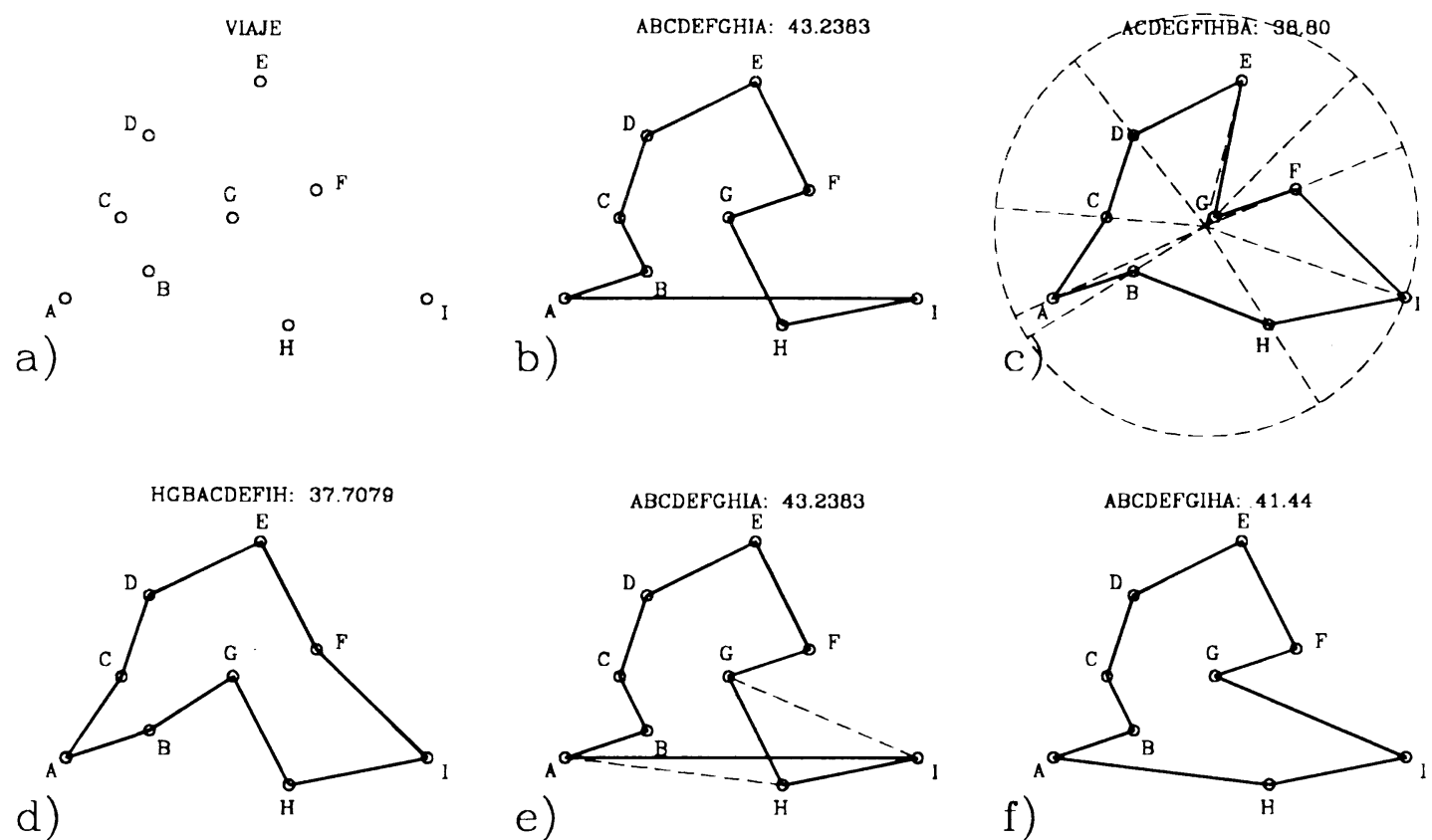

Fig.3.- El problema del viajante de comercio: De izquierda a derecha, y de arriba abajo: a, un caso del problema del viajante; $b$, un circuito generado al azar; $c$, un circuito generado con el apoyo de una circunferencia con centro en el centro de gravedad de las ciudades; $d$, itinerario óptimo; $e$, un circuito no óptimo debido al cruce de dos trayectos; $f$, circuito -mejor que el anteriorobtenido mediante la regla "eliminar cruces".

Tabla 2

Tiempo de Cálculo para la resolución del problema del viajante

\begin{tabular}{ccc|}
$\begin{array}{ccc}\text { Ciudades } \\
n\end{array}$ & $\begin{array}{c}\text { Recorridos } \\
0,5 \cdot(n-1) !\end{array}$ & Tiempo de cálculo \\
\hline 3 & 1 & 1 segundo \\
6 & 60 & 1 minuto \\
9 & 20.160 & 5 horas \\
12 & 19.958 .400 & 231 días \\
15 & 43.589 .145 .600 & 13 siglos \\
18 & $1,77 \times 10^{14}$ & 5.640 milenios \\
\hline
\end{tabular}

En efecto, cualquier orden de las ciudades constituye un recorrido posible que tiene una distancia bien definida (Fig. 3b). Además, el número total de itinerarios posibles es finitoy sólodepende del número de ciudades (exactamente $0,5 \times(\mathrm{n}-1)$ !, siendo $n$ el número de ciudades). Por tanto el problema se resuelve calculando la distancia de cada uno de los recorridos y eligiendo el de longitud mínima, que es la solución buscada (Fig. 3d).

Este método de resolución, denominado búsqueda exhaustiva, no requiere, en consecuencia, más que un número finito de operaciones. Este primer resultado es importante, pues se conoce ya un buen númerode problemas para los que se ha demostrado que ningún método podrá resolverlos si se exige esta última condición.

En cualquier otro problema de optimación, basta con que cada solución candidata pueda representarse como una lista finita de símbolos y que el conjunto de todas ellas lo sea también, para que pueda asegurarse que la búsqueda exhaustiva lo resolverá

\subsection{Intratabilidad}

Desafortunadamente, que un problema sea resoluble no asegura que su resolución esté a nuestro alcance, pues a pesar del número finito de operaciones necesarias para buscar la solución, este número puede ser tan grande que el tiempo de cálculo necesario supere a la antigüedad de nuestra especie o a la edad estimada del universo. Para ver esto basta considerar distintos casos concretos del problema del viajante, (Tabla 2); en la tercera columna figura el tiempo de cálculo del itinerario óptimo, bajo el supuesto de que los cálculos de la longitud de cada uno de ellos pueden hacerse en un segundo por circuito.

Lo significativo de la tabla reside en que a un viajante de comercio de poco le sirve saber que $s u$ problema tiene solución: si cada viaje entre dos ciudades le llevara una media de un día, y si tuviera que visitẵ doce ciudades o más, sería mejor recorrerlas al azar, pữes de todos modos tardaría menos en recorrerlas (incluso del peor modo 
posible) que en calcular cuál hubiera sido el mejor de los itinerarios. De este modo, aunque este problema puede ser resuelto, en la mayoría de los casos prácticos de poco sirve saberlo. Para denominar esta situación se dice que, hoy por hoy, el problema del viajante de comercio es intratable, lo que, a efectos prácticos, es tanto como decir que es irresoluble. Formalmente, el tiempo de cálculo requerido por la búsqueda exhaustiva no guarda proporción con el tamaño algorítmico del problema, medido de manera informal por el número $n$ de ciudades. Es decir, el algoritmo no es polinómico (de hecho, se trata de un algoritmo fuertemente exponencial, aunque su convergencia esté asegurada).

Pero, en rigor, nadie ha sido capaz de demostrar que sea imposible encontrar en el futuro algún otro método de resolución que no consuma cantidades tan fantásticamente grandes de tiempo. Quizá se deba a nuestra torpeza el no haberlo encontrado hasta ahora. Sin embargo, sólo existe una pequeña esperanza de que ello sea posible, una esperanza leve que se apoya en el hecho de que,en ciertos casos, el problema del viajante de comercio no sólo se resuelve, sino que se resuelve de un plumazo.

Considérese, por ejemplo, el caso particular en que las ciudades se disponen a lo largo de una circunferencia, (Fig.4). Entonces resulta claro que el polígono inscrito de la figura es la solución óptima. En este caso el tiempo de solución es proporcional al número de ciudades $\mathrm{y}$, en general, el tiempo de cálculo necesario es siempre menor que el invertido en recorrerlas, de manera que el viajante de comercio puede realizar sus visitas del mejor modo.

Un cierto número de casos semejántes son conocidos, pero sin embargo, a pesar de perseverantes esfuerzos de investigadores de reconocido talento, nadie ha dado con un método de resolución del problemageneral que sea práctico o tratable (incluso contando con la ayuda de velocísimas máquinas de cálculo). Por ello, la creencia más extendida es que el problema es verdaderamente intratable (aunque nadie lo haya demostrado).
Muchos otros problemas matemáticos pertenecen a esta categoría, y son conocidos como problemas NP-completos. El mayor avance en su estudio consiste en haber demostrado que si uno de ellos pudiera ser resuelto de un modo práctico o tratable, a todos los demás les sucedería lo mismo.

\subsection{Algoritmos de aproximación}

¿Qué puede hacerse con un problema aparentemente intratable si verdaderamente se necesita la solución? Pensemos en un viajante de comercio con doce ciudades que visitar: si contara con un método de calcular, no ya la solución óptima, sino alguna bastante buena, y el cálculo con tal método no le llevara más de una docena de minutos, podría darse por satisfecho: a fin de cuentas no haría su viaje por el peor de los recorridos posibles y tampoco gastaría una cantidad desproporcionada de tiempo en calcular el mejor de todos.

Debe quedar claro, no obstante, que los algoritmos que calculan buenas soluciones no son algoritmos de resolución; su ventaja reside en que se trata de algoritmos polinómicos y que, por tanto, ofrecerán una aproximación en un tiempo razonablemente proporcionado a la dificultad algorítmica del caso abordado. Para cada problema genérico existen numerosos algoritmos semejantes e, incluso, métodos genéricos de diseñarlos.

Un método común consiste en partir de un caso particular en que la solución óptimaes trivialmente calculable mediante un algoritmo específico: al aplicar éste a otros casos genéricos se obtiene ya una aproximación, que funcionará tanto mejor en tanto el casogenérico no se aparte mucho del caso particular fácilmente resoluble.

Si, por ejemplo, las ciudades no están dispuestas a lo largo de una circunferencia, pero tampoco se apartan en exceso, puede actuarse como si lo estuvieran: el recorrido no será el peor y el costo de obtenerlo es razonablemente pequeño
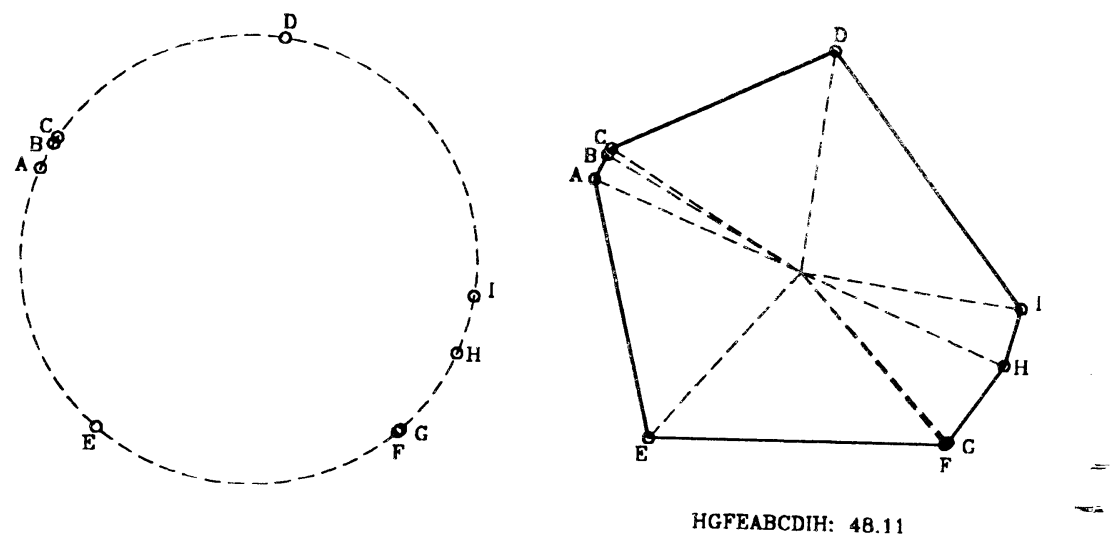

(c) Consejo Superior de Investigaciones Cienf.-Un caso "facil" del problema del viajante. 
$\mathrm{y}$, en cualquier caso, proporcionado al número de ciudades (Fig. 3c). En el sencillo ejemplo ilustrado, de hecho, la solución obtenida se compara bien con la solución óptima. Que el circuito así obtenido no es el peor, se sabe por criterios de carácter local: en el problema del viajante, un circuito en que dos trayectos se cruzan no es óptimo, por el contrario, cualquier circuito libre de tales cruces no es pésimo (Fig. 3, e y f).

Con criterios de optimidad semejantes a éste de los cruces, pueden construirse otros algoritmos de aproximación que permiten mejorar soluciones obtenidas al azar. Tales criterios o reglas heurísticas dependen de la naturaleza particular de cada problemaabordado y representan bastante bien el conocimiento acumulado acerca de la naturaleza de la solución, aunque ésta permanezca desconocida. Nótese, además, que los algoritmos de aproximación suelen ser innecesarios si no se desea una precisión desmedida: $l o$ importante son las reglas; y éstas pueden aplicarse intuitivamente para obtener buenos resultados, tal y como en el circuito para una taladradora automática mostrado en la (Fig. 5), a la izquierda, que fue diseñado manualmente. Para este caso, uno de los algoritmos heurísticos más eficientes entre los conocidos (Lin et Kernighan, 1973), tan solo consiguió reducir la longitud obtenida a mano una pizca, un $2 \%$, véase la (Fig. 5), a la derecha. La única ventaja de los algoritmos, en tanto, procedimientos formales paso a paso, es que pueden ser ejecutados mediante máquinas, de un modo ciego y automático.

\section{Recocido simulado}

En contraposición a los algoritmos heurísticos específicos de cada problema, el recocido simulado es un método polinómico de aproximación bastante universal, que permite mejorar significativamente una solución elegida al azar. Para mejor ilustrar el algoritmo de recocido describiré sucintamente su aplicación al problema del viajante de comercio, aunque su fundamentación matemática ha sido objeto de atención por diversos investigadores (véase, por ejemplo, Hajek, 1988).

\subsection{Representación}

Cada circuito es un orden de las ciudades (Fig. 3). Como se trata de un circuito cerrado, cuál sea la ciudad en la que se inicia el viaje carece de importanciay, en consecuencia, puede elegirse una de ellas arbitrariamente como primera ciudad.

Un circuito puede representarse de muchas maneras: la lista ordenada de los nombres de las ciudades, su dibujo, etc. Pero para lo que aquí interesa, considérese la siguiente tabla:

ABCDEFGHIA: longitud 43,2383

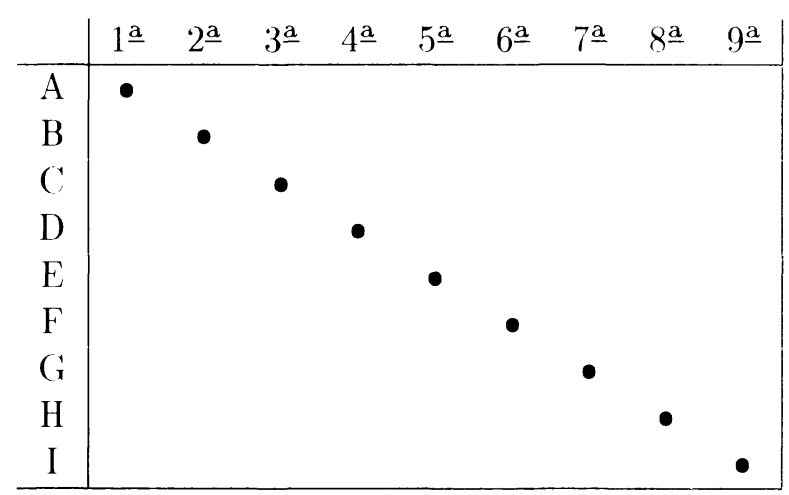

Cada casilla marcada representa el hecho de que la ciudad correspondiente a su fila ocupa en el itinerario el lugar correspondiente a su columna. Para construir un circuito en esta representación, basta con señalar una casilla de la tabla por cada ciudad, teniendo en cuenta que la casilla correspondiente a la ciudad origen es fija y que sólo puede marcarse una casilla en cada fila y en cada columna. De este modo, tablas como ésta son circuitos, cuyas longitudes pueden calcularse.
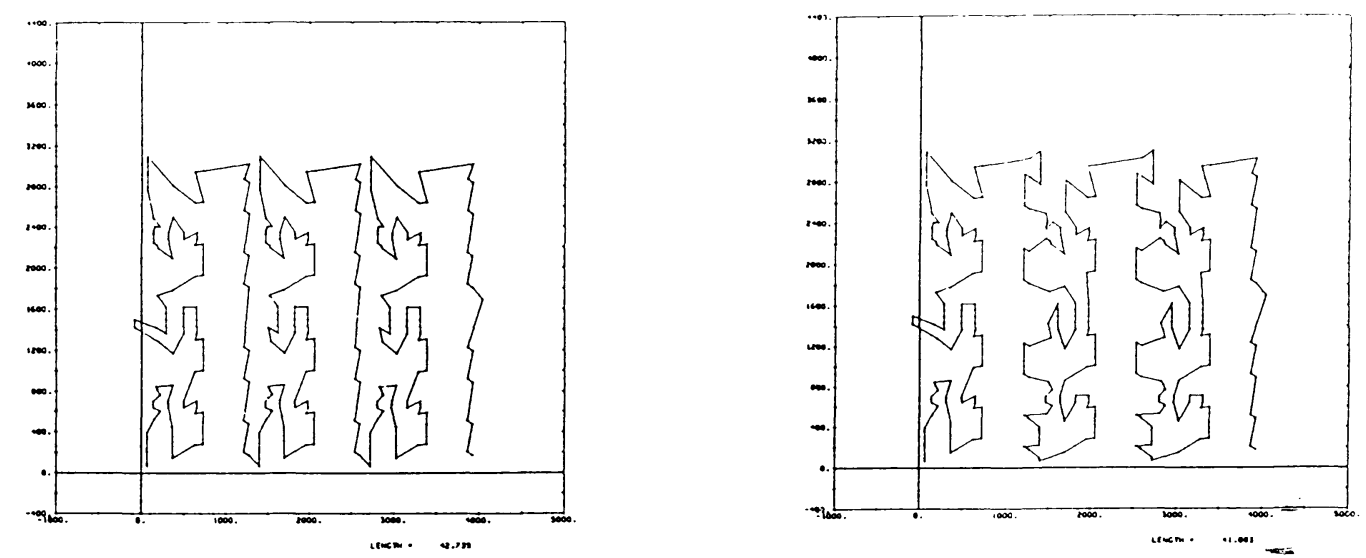

Fig.S.-Aproximación manual y automática : A la izquierda, una solución obtenida a mano para el itinerario de una máquinaherramienta $(L=42,7)$; a la derecha, la aproximación de un algoritmo heurístico $(L=41,8)$. 

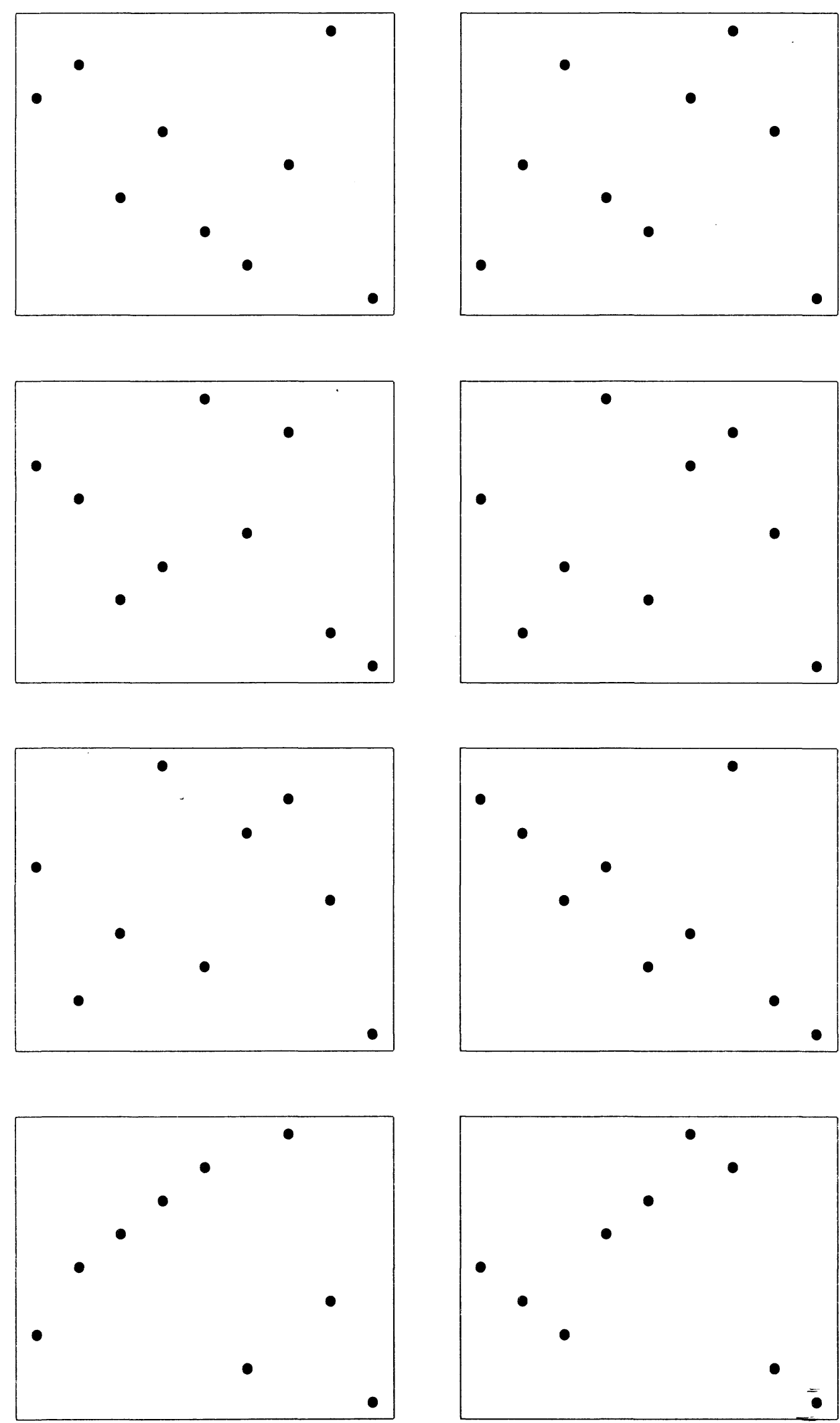

Fig.6.- Distintos circuitos elegidos al azar representados mediante tablas.

(c) Consejo Superior de Investigaciones Científicas 
Para elegir un circuito al azar basta elegir para cada columna una ciudad no elegida todavía y marcar la casilla correspondiente. Si se eligen varios recorridos de este modo, el aspecto de la tabla va cambiando aleatoriamente, de un modo análogoa como las partículas de un gas caliente van cambiando de posición en el recipiente que lo contiene (Fig. 6). Cada casilla marcada representa, por así decir, una molécula de un gas imaginario. Desde luego, se trataría de un gas bastante raro, pues sus moléculas están compelidas a no compartir ni filas ni columnas, resultando una analogía no del todo satisfactoria.

Este gas imaginario puede ser forzado a solidificarse, si se hace descender su temperatura igualmente imaginaria. Sus moléculas acabarían en posiciones fijas, aquéllas que pudieran alcanzar antes de perder toda movibilidad. Si además el enfriamiento fuese suficientemente lento, la distribución de las moléculas correspondería al valor mínimo de su energía cinética, cuyo valor es igual al de la longitud del circuito, por la analogía. A lo largo del proceso, la configuración de las moléculas del gas (o el orden de las ciudades) ha cambiado en apariencia al azar. Pero no se trata de un azar puro: en un gas real son los choques de moléculas los que provocan los cambios, de manera que las sucesivas configuraciones están en realidad correlacionadas, aunque sólo estemos en condiciones de dar una imagen imprecisa, estadística, de tal correlación, y aunque en múltiples aplicaciones tales correlaciones puedan ignorarse, al resultar despreciables.

La estadística de Maxwell-Boltzmann puede y debe ser empleada para hacer funcionar este imaginario frigorífico:

1.Puede, porque en su deducción no se prescribe ninguna característica particular que deba cumplir lafunción energía, de manera que la longitud puede ocupar su lugar.

2.Debe ser empleada, además, para introducir en el azar el sesgo de las correlaciones y fluctuaciones del proceso en un gas real, única forma de asegurar que la configuración estable fundamental pueda ser alcanzada.

\subsection{Transiciones}

Para simular el comportamiento de este gas a temperatura constante la técnica se resume en el algoritmo de Metropolis. Esencialmente consiste éste en una sucesión de transiciones o jugadas producidas en dos pasos:

1.Se provoca un cambio aleatorio en la configuración, y se evalúa el cambio correspondiente en la energía, y

2.Se acepta o rechaza el cambio, de acuerdo con la distribución de Boltzmann.

En el caso de los itinerarios del viajante hay varias maneras posibles de transformar aleatoriamente un circuito dado.
La más simple es intercambiar dos ciudades elegidas al azar, tales como la A y la C en la Fig. 7. La diferencia entre ambas longitudes o energías, $L$ ' - $L$, podrá ser positiva 0 negativa. Esto completa el primer paso del cálculo de la transición. Para elegir al azar entre dos opciones, el recurso habitual es lanzar una moneda al aire. En un programa de ordenador, la moneda se sustituye por series de números pseudoaleatorios cuyos valores están comprendidos entre cero y uno. Cada nuevo número de la serie debe tener un valor sin correlación con el anterior (las series que se suelen vender junto a los ordenadores no suelen cumplir esta condición; para series apropiadas, véase Press et alii, 1988:205-213). Si A representa a cada número de una de estas series, la fórmula

$$
i=1+\mathrm{E}(\mathrm{n} \times A) \quad A \in(0,1)
$$

permite elegir una ciudad $i$ al azar entre las $n$ del conjunto $-E(x)$ es la parte entera de $x$.

Para decidir si la nueva configuración se acepta o se rechaza, existen varias reglas de selección posibles. La originalmente utilizada en el algoritmo de Metropolis tiene, a su vez, dos partes:

1. Si el cambio en la energía, $\Delta L$, es negativo, es decir, si la nueva configuración obtenida al azar disminuye la energía del gas, se acepta siempre esta última.

2. Si, por el contrario, dicha diferencia es positiva se acepta la nueva configuración (de mayor longitud o energía) con una probabilidad dada por la siguiente fórmula:

$$
p(\Delta L)=\exp (-\Delta L / T)=\frac{1}{\exp (\Delta L / T)}
$$

En estafórmula, $T$ cumple el mismopapel que la temperatura en un gas real, pero ciertamente se trata de una cantidad análoga a la temperatura y no la temperatura habitual. Si esta última es una medida indirecta de la cantidad media de energía contenida en un gas real, en nuestro caso se relaciona con el valor medio de la "cantidad de viaje", por así decir, contenida en la secuencia de circuitos que vamos obteniendo mediante sucesivas jugadas.

La regla de selección anterior se denomina oráculo de Metropolis y se resume en:

$p(\Delta \mathrm{L})= \begin{cases}1 & \text { si } \Delta L=L^{\prime}-L<0 \\ \exp (-\Delta L / T) & \text { en otro caso }\end{cases}$

Su significado puede resumirse observando que, si el circuito nuevo es mejor que el antiguo, se acepta siempre el cambio, mientras que en caso contrario sólo se acepta en ocasiones.

Cuando la jugada conduce a un aumento del valor de la función, es decir, cuando se trata de elegir un circuito más 
largo, merece la penailustrar algunas situaciones siguiendo la evolución del recocido:

1. Si $T \approx 100 \Delta L$ entonces:

$p(\Delta L)=\frac{1}{\exp (\Delta L / T)} \approx \frac{1}{\exp (\Delta L / 100 \Delta L)}=\frac{1}{\exp (0,01)}=\frac{1}{1,01}=$ $=0,99$

En la situación inicial la temperatura es alta, de hecho, muy alta, y como corresponde a un gas caliente, la probabilidad de que cualquier nueva configuración sea aceptada es prácticamente del cien por cien.

\section{Si $T \approx \Delta L$ entonces:}

$p(\Delta L)=\frac{1}{\exp (\Delta L / T)} \approx \frac{1}{\exp (\Delta L / \Delta L)}=\frac{1}{\exp (1)}=\frac{1}{2,72}=$ $=0,37$

Según latemperatura disminuye, el panorama va cambiando paulatinamente. Para temperaturas del mismo orden de magnitud que la diferencia de longitudes, la probabilidad de que el aumento sea aceptado baja hasta el 37 por ciento.

3. Si $T \approx 0,01 \Delta L$ entonces:

$p(\Delta L)=\frac{1}{\exp (\Delta L / T)} \approx \frac{1}{\exp (\Delta L / 0,01 \Delta L)}=\frac{1}{\exp (100)}=$ $=\frac{1}{2,69 \times 10^{43}} \approx 0$

Con temperaturas mucho menores que la diferencia de longitud, la probabilidad se vuelve prácticamente nula. A bajas temperaturas, el itinerario apenas cambiará y puede decirse que se ha solidificado, convirtiéndose en la solución mejor que el algoritmo puede ofrecer
¿Cómo puede aceptarse una configuración peor con una probabilidad dada? De nuevo la técnica se apoya en series de números pseudoaleatorios. Formalmente, para realizar una elección con probabilidad $p(\Delta L)$, se elige el siguiente número de la serie $A$, si $A<p(A L)$ la configuración se acepta; en otro caso, se rechaza. El procedimiento es similar a escoger un punto al azar en un rectángulo: si la probabilidad de aceptar la nueva configuración debe ser, digamos, de 0,6, la configuración se acepta si el punto cae en el $60 \%$ de la superficie del rectángulo que queda a la izquierda, que es, por así decir, la zona de aceptación para dicha probabilidad. Si la serie de números es de buena calidad, el comportamiento del gas imaginario quedará bien simulado. Pero siempre será simulado del mismo modo (salvo que se cambie de serie de números aleatorios), lo que no sucedería si se usara una moneda. El comportamiento del algoritmo está, por tanto, determinado, como cabía esperar de un programa de ordenador.

\subsection{Disminución de la temperatura}

El recocido simulado consiste en ciclos de cálculo a temperatura constante y en una disminución paulatina de la temperatura entre ellos, (Tabla 3). En cada ciclo (paso 3) se efectúa un número de transformaciones proporcionado al número de variables o grados de libertad del sistema en estudio,tal como el número de ciudades en el caso del viajante.

Tabla 3

Algoritmo de recocido

1. Verificar y evaluar la solución inicial.
2. Dar valor a la temperatura inicial.
3. Para cada ciclo:
Para cada transición dentro del ciclo (algoritmo de
Metropolis):
1. Obtener una nueva configuración al azar.
2. Aceptarla o rechazarla según el oráculo de Metropolis
o regla de selección coherente con la distribución de
Boltzmann.
Disminuir la temperatura.
(b) Mostar la configuración final.

$$
L^{\prime}=45,19
$$
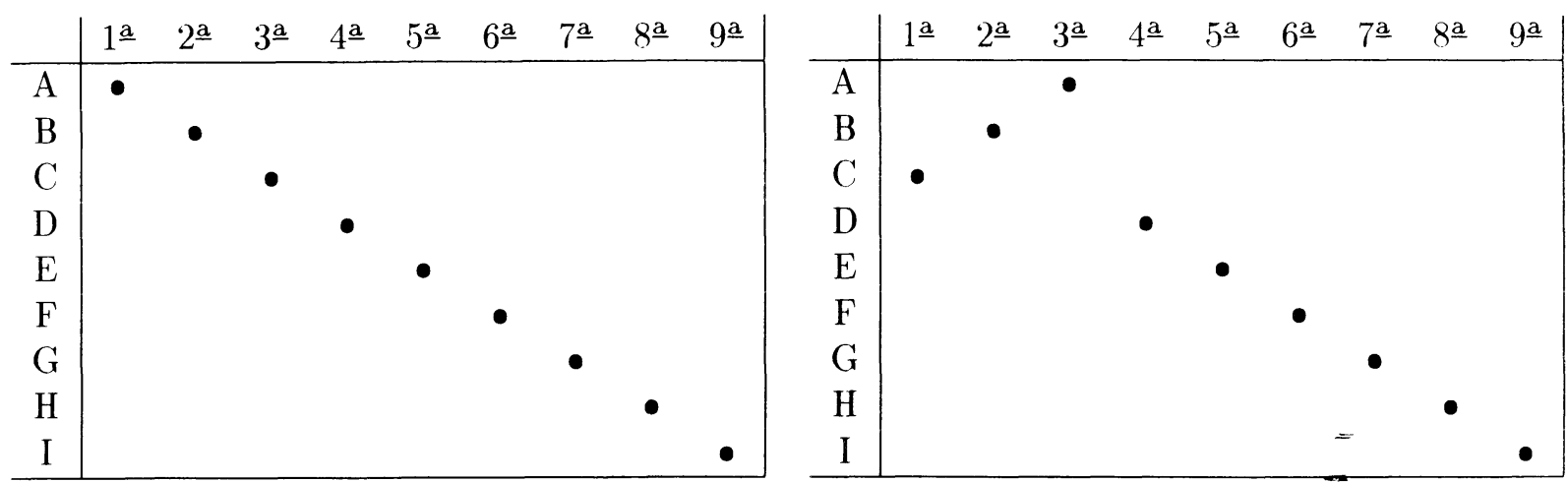

Fig.7.- Transición entre dos circuitos. 
El generador ilustrado para el problema del viajante, es decir, permutar dos ciudades en el itinerario, p r od u ce pocos cambios cada vez (en el paso 3.a.1). Pero puede demostrarse con facilidad que con un número de transformaciones de este tipo, finito y acotado por un polinomio del número de ciudades, existe una cierta probabilidad, aunque pequeña, de alcanzar cualquier configuración de las posibles. En general, los generadores para cualquier problema sólo deben cumplir dos condiciones simples:

1. Realizar la transformación al azar, es decir, con probabilidad uniforme entre todas las configuraciones accesibles o vecinas.

2. Ser capaz de alcanzar cualquier otra configuración en un número finito de transformaciones.

Puesto que normalmente la configuración inicial al principio de un ciclo no será una configuración de las probables a la temperatura correspondiente, el número de transformaciones por ciclo debe ser generoso, a fin de que pueda alcanzarse una representación estable del comportamiento del sistema para esa temperatura, es decir, el sistema debe tener oportunidad de relajarse. Una cifra común en el problema del viajante es realizar tantas jugadas como cien veces el número de ciudades, aunque para simulaciones de sistemas termodinámicos, se emplean cifras muy superiores, del orden de 10.000 transiciones por cada grado de libertad.

La temperatura inicial debe ser mucho mayor que el valor medio de la energía de una muestra de configuraciones elegida al azar (paso 2). Esto es necesario para simular el comportamiento típico de un gas caliente.

Para disminuir la temperatura de ciclo en ciclo (paso 3.b), puede utilizarse un factor próximo a la unidad pero menor que ella, tal como 0,9 ó 0,95. De manera que la temperatura del ciclo $i$ viene dada por fórmulas del tipo $T_{i}=0,9 \times T_{o}$, en donde $T_{\mathrm{o}}$ es la temperatura inicial.

No resulta difícil mostrar que con estas reglas se alcanzan temperaturas extremadamente bajas en un número finito
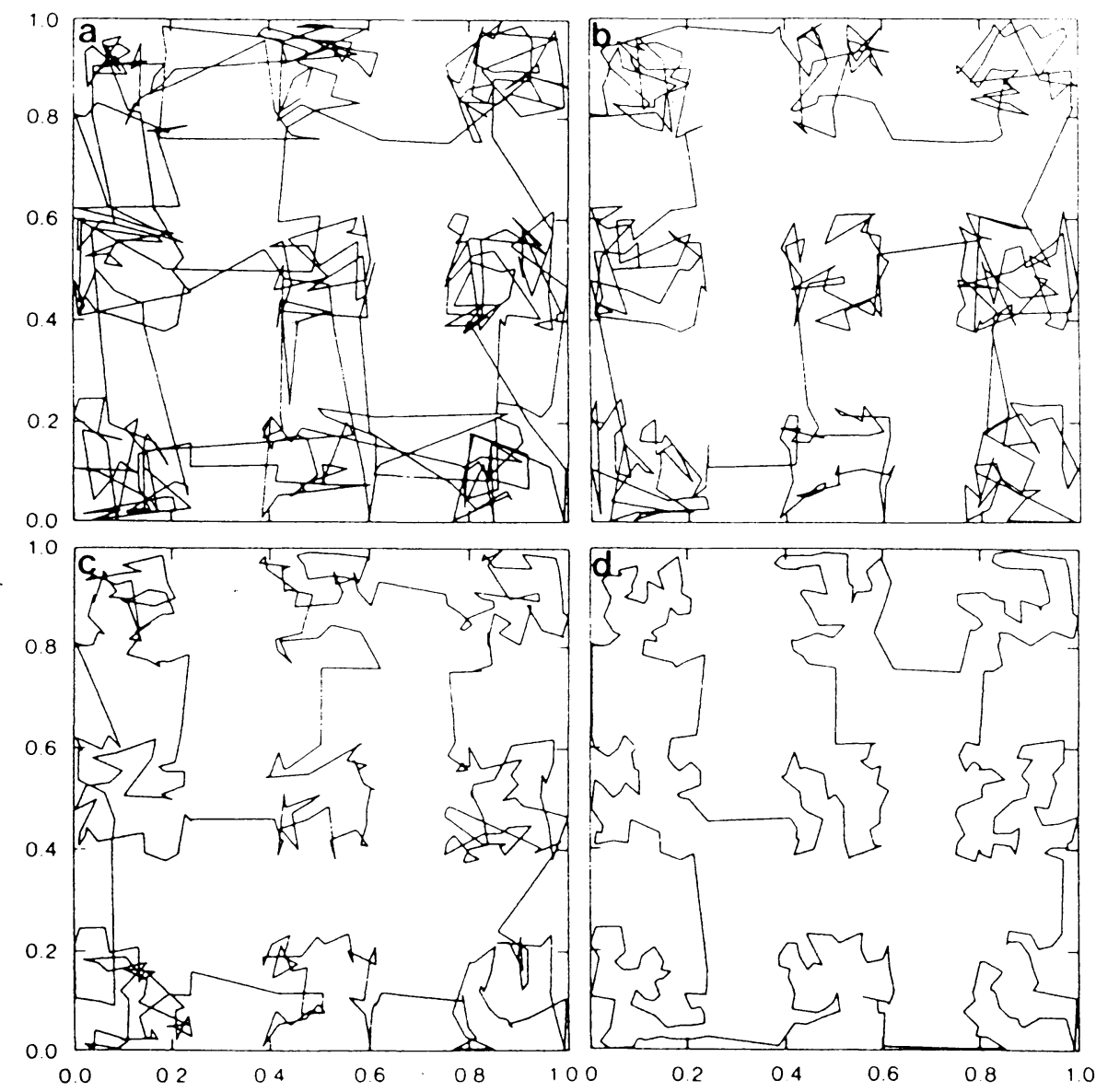

Fig. 9. Results at four temperatures for a clustered $4(0)$-cily traveling salesman problem. The points are uniformly distributed in nine regions (a) $T=1.2, \alpha=2.0567$ : (t) $T=0.8$. $\alpha=1.515:$ (c) $T=0.4=1.055:$ (d) $T=0.0 . \alpha=0.7834$

Fig.8.- Aproximación a un caso del viajante. 
de ciclos, esencialmente independiente del problema en estudio, y del orden de un centenar o dos. Con este esquema, el numero de pasos de cálculo, aunque grande, es proporcionado al número de variables que definen la configuración y el algoritmo resultante es tratable, lo que ha permitido aproximar casos del problema del viajante con numerosas ciudades (Kirkpatrick et alii, 1983), cuya resolución exacta exigiría tiempos inconcebibles (Fig. 8).

El algoritmo es relativamente lento si se compara con otros algoritmos de aproximación más cercanos a la heurística de cada problema, pero no suele equivocarse en mucho. En cierto sentido, esta lentitud es la contrapartida a disponer de un algoritmo sencillo, universal y robusto.

Una pregunta que surge ocasionalmente es la siguiente: $\mathrm{si}$ el recocido funciona escogiendo siempre una alternativa mejor, pero a veces también una peor ¿no resultará más eficiente rechazar siempre esta última? Tal algoritmo de hecho existe con caráctergeneral (se llama mejora iterativa y puede compararse con el temple del acero). La ventaja que el recocido tiene sobre él es, precisamente, no encaminarse codiciosamente hacia la solución mínima más cercana, que bien pudiera no ser la mínima absoluta. La situación es bien conocida: en la Fig. 9 se muestran dos isómeros del mismo alcohol que tienen propiedades diferentes y también diferente energía de formación. Ambos son estables y la transición del isómero $2 \mathrm{al} 1$ no se produce espontáneamente. Esto se debe a que no se trata de configuraciones vecinas: para que la transición tenga lugar debe aportarse energía adicional hasta escalar a la divisoria de las cuencas, y luego volver a bajar (véase Schrödinger, 1944). Con sus "saltos hacia arriba" el recocido tiene alguna probabilidad de escapar de valles, que como el 2 , no contienen la configuración estable fundamental.

\section{Cerchas isostáticas óptimas}

Lo visto hasta aquí es ciencia y técnica consolidada que ha sido empleada con éxito para la resolución aproximada de problemas en muy diversas disciplinas. En el campo concreto de la teoría de estructuras, también se ha empleado con éxito en problemas muy diversos; hasta la fecha en cuatro (Vázquez, 1994):

1. Análisis de losas mediante la teoría de líneas de cedencia

2. Búsqueda de formas óptimas de cúpulas de revolución cargadas axisimétricamente.

3. Dimensionado óptimo de cerchas hiperestáticas cuya forma general está dada.

4. Búsqueda de formas óptimas de cerchas estáticamente determinadas.

El algoritmo empleado en este último caso se examina con algún detalle a continuación.

Para lo que aquí interesa, puede entenderse una cercha como aquella estructura plana que sostiene pesos situados en puntos determinados, apoyándose a su vez en otros puntos fijos (Fig. 10). Unos puntos y otros, también llamados nudos, están unidos a través de barras y por intermedio de otros puntos. Los puntos de apoyo o de suspensión de pesos son fijos, cualquier otropunto (destinado únicamente a unir barras) es variable.

Un modelo simple y realista para el análisis de estas estructuras resulta al considerar sólo las fuerzas de tracción y compresión de las barras. En tal caso, la cercha se dice<smiles>CCCO</smiles><smiles>CC(C)O</smiles>

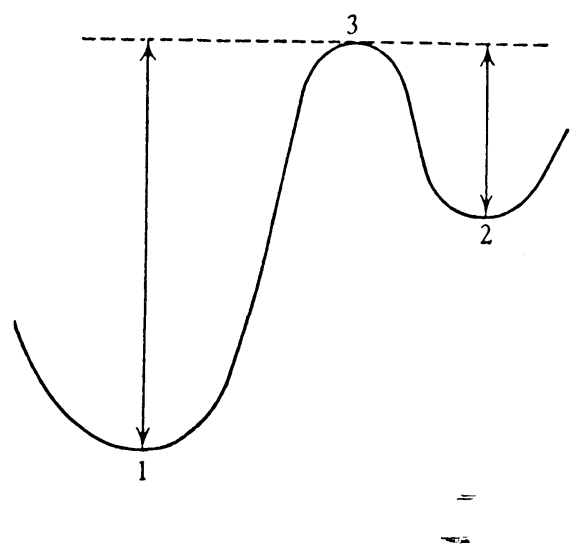

Fig.9.- Metaestabilidad: A la izquierda, dos configuraciones del propanol; a la derecha, su situación en el "paisaje energético". 

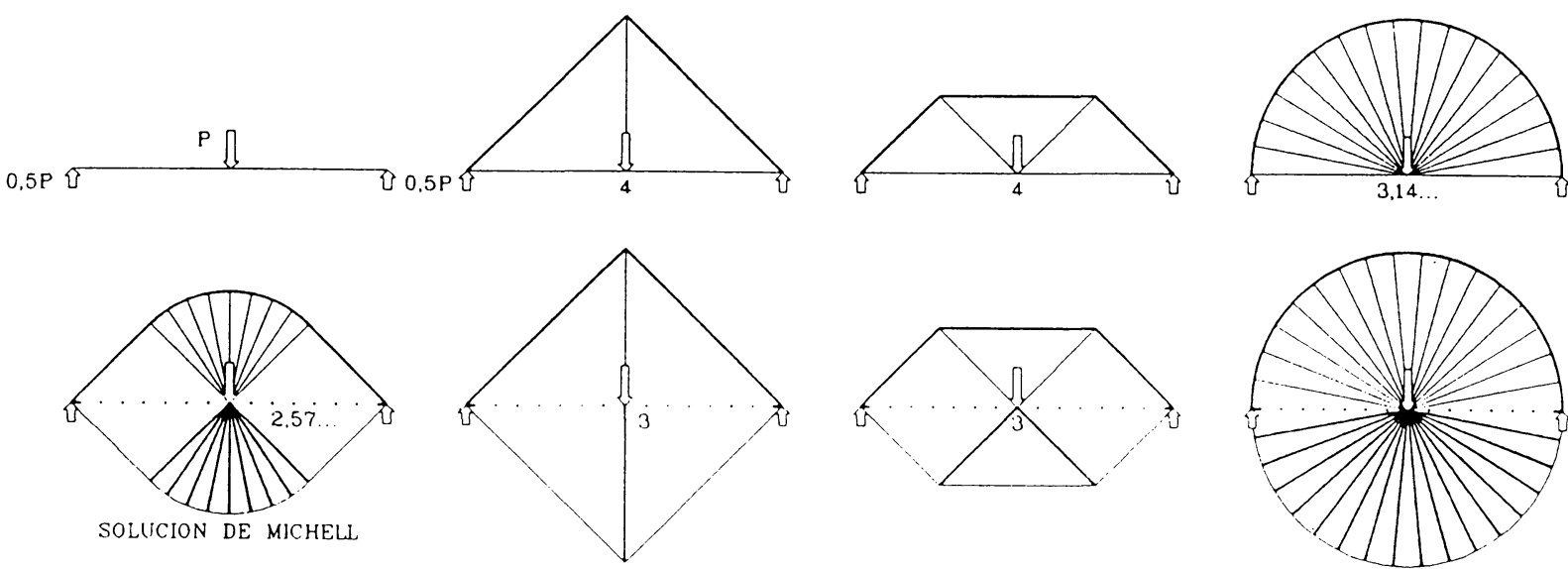

Fig.10.- Distintas cerchas para sostener un único peso: las barras regruesadas están comprimidas. Las cifras que acompañan cada solución son proporcionales a la cantidad de material.

isostática internamente si, dependiendo de la posición de los puntos variables y de la conexión de los distintos puntos por barras, pueden determinarse las fuerzas que los pesos originan en éstas, incluso antes de averiguar la cantidad de material que debe disponerse en cada una. Esta última puede determinarse después según la resistencia del material con que se construye y, por tanto, puede calcularse la cantidad de material total que es necesaria.

En consecuencia, y a condición de que la cercha sea isostática internamente, para cada forma siempre puede encontrarse la cantidad mínima de material que se necesita para que la cercha resista con seguridad los pesos. Todas las formas isostáticas pueden por tanto ser seguras, pero a costa de cierto material, variable con la forma.

En el espíritu de científicos como Maxwell o Michell debió existir la intuición o la convicción de que la economía debería tener que ver con algo tangible, como por ejemplo ahorrar material, más que con magnitudes intangibles e imprecisas como son los precios, que es de lo que charlan casi todos los economistas. Sorprendentemente, tal forma de pensar, lade Maxwell o Michell, perdura vigorosamente, a pesar de la cultura económica dominante hoy en día. No hace falta ir muy lejos para encontrar ejemplos: baste citar los escritos de Félix Candela (1985), investido doctor honoris causa por la Universidad Politécnica de Madrid recientemente.

Sea como fuere, Michell (1904), hace poco más de nueve décadas, llamó la atención sobre el hecho de que no era lo mismo cualquier forma para una cercha isostática (aunque tal forma siempre podría resultar aceptable en términos de resistencia) e investigó de qué modo podría determinarse aquella forma óptima que requeriría el mínimo coste (Fig. 11).

Como función objetivo, Michell adoptó el peso total de la cercha. Si sólo se usa un material, el peso y el volumen son
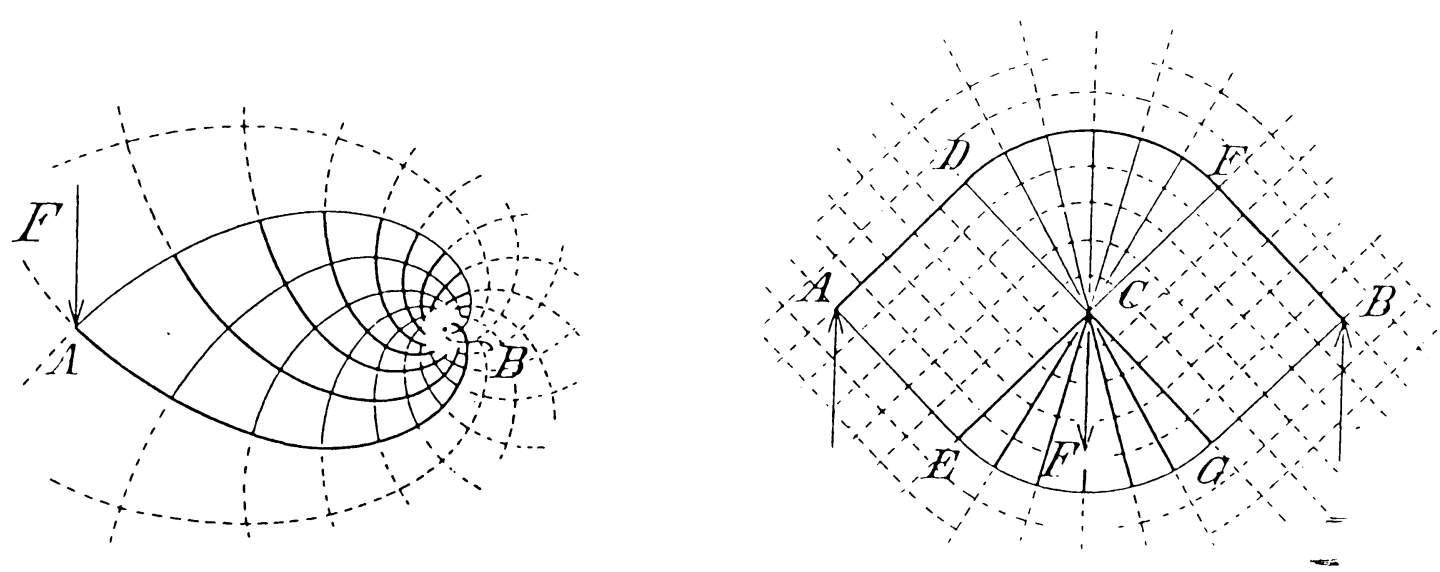

Fig.11.- Soluciones óptimas de Michell: A la izquierda, para sostener un peso en vuelo; a la derecha, entre dos apoyos. 
proporcionales (véase, por ejemplo, Quintas, 1992). En tal caso, multiplicando el volumen por la resistencia del material, se obtiene otra cantidad proporcional, que tiene la ventaja de permitir la comparación en términos estructurales de cerchas con forma y material distintos, y que, en lo sucesivo, denominaré volumen estructural. El volumen estructural, el volumen a secas o el peso representan el mismo objetivo, pero medido a escalas distintas ${ }^{1}$.

\subsection{Dificultad del problema}

A pesar de la antigüedad de su planteo, el problema de obtener la cercha isostática óptima sólo ha sido resuelto para muy pocas condiciones de carga y apoyo. Además, no se conoce (que yo sepa) ningún algoritmo capaz de aproximar una solución considerando tanto variaciones en la posición de los nudos como en la conexión de las barras. No en vano este problema ha sido calificado como la más difícil de las tareas de optimación estructural (Vanderplaats, 1984).

Para sopesar su dificultad, considérese un ejemplo sencillo, uno de los resueltos por Michell (Figs. 10 y 11). La cercha debe conectar mediante sus barras el punto de suspensión del peso (punto $\mathrm{C}$ en la Fig. 11) con los puntos de apoyo (A yB). Para ello puede emplearse cualquier número de nudos adicionales. Ya se comprende que para asegurar que la solución pueda describirse con un número finito de variables hay que imponer un límite superior al número total de puntos (denominado $M$ en adelante).

Además, para que la estructura sea isostática internamente el número de barras y condiciones de apoyo debe ser necesariamente igual al doble del número de nudos. Esta bien conocida condición determina el número total de barras en función del número de nudos (no puede haber ni más ni menos) pero no sus conexiones. La condición es sólo necesaria, de manera que, aún cumpliéndola, laforma de la cercha puede resultar finalmente inestable y, por tanto, inviable.

En definitiva, existen trestipos de variables en la definición de cada una de las cerchas posibles:
1. El número total de nudos de la cercha, que ha de ser menor o igual que el límite impuesto, y obviamente mayor o igual que el número de puntos fijos.

2. La posición en el plano de cada uno de los puntos variables, añadidos a los fijos.

3. La pareja de nudos que quedan unidos por cada una de las barras estrictamente necesarias para constituir una cercha isostática.

Cada cercha así definida es una configuración, una solución candidata. En este caso la definición de la configuración resulta peculiar pues el valor de uno de sus parámetros, el número total de nudos, determina el número de los restantes.

Conviene resaltar aquí el parentesco indudable entre este problema y el del viajante de comercio: en ambos se trata de buscar una forma óptima respecto a una magnitud que depende de cómo se conecte un conjunto de puntos en el plano. El carácter combinatorio del espacio de soluciones candidatas resultaevidente. Asíque una conjetura plausible es que este problema será al menos tan difícil como el del viajante.

Pero hay una diferencia notable. Mientras que en el problema del viajante cualquier orden de las ciudades es una solución candidata, aquí no vale cualquier forma con las condiciones enunciadas, pues las soluciones candidatas deben ser realmente isostáticas. Qué se cumpla esta condición sólo puede comprobarse una vez la forma ha sido elegida. En consecuencia, la exploración debe abarcar más formas que las estrictamente seguras y estables.

Para recocer este problema, sin embargo, el esquema es esencialmente el mismo: hay que construir generadores capaces de alterar cada una de las variables que definen una configuración, a fin de obtener otra al azar.

Antes de examinarlos, nótese una característica especial de los nudos variables que permite reducir algo el espacio de búsqueda de la solución. Sobre estos nudos no actúa ninguna fuerza exterior. Debido a ello puede demostrarse

1. La magnitud aquí denominada "volumen estructural", $W$, se calcula como el producto del volumen de material, $V$, por la tensión nominal del mismo, f. Si el dimensionado es estricto (en tracción y en compresión), $W$ también es igual a la suma extendida a todas las barras del producto del valor absoluto de la solicitación, $N$, por la longitud, $L$, es decir $\Sigma$ abs $(N)$. L. Esta última magnitudfigura en lostrabajospioneros deMaxwell y Michell. También fue objeto de estudio, entre otros, por Miguel (1974) quien la denominó "trabajo estructural", pero la expresión no prosperó. Aroca y Cervera, (1989 y 1993) la denominaron, finalmente, "cantidad de estructura", $C$. Sin embargo, recientemente, Aroca (1994) está empleando, en sus clases en la Escuela de Arquitectura de Madrid, "volumen estructural" para $C=\Sigma$ abs $(N)$. L. Pormipartepropongo, provisionalmente, conservar "cantidad de estructura" para la suma de abs $(N)$. $L$ y volumen estructural paraf $V$ que es la que se emplea en el texto. La cantidad de estructura $C$ es idénticamente igual al volumen estructural $W$ si eldimensionado es estricto artificio, este último, extremadamente útil para establecer una teoría de diseño de estructuras (véase un conciso y competente resumen en Cervera, 1993). En consecuencia, cuando el contexto despeje cualquier confusión, $C$ podría también denominarse "volumen estricto" y $W$ "volumen", simplemente. El primero es siempre una cota inferior significativa del segundo. 
con facilidad que si a un nudo variable sólo acometen dos barras, el propio nudo y ambas barras pueden suprimirse y el volumen estructural resultante será igual o menor, debido a que el esfuerzo en ambas barras será nulo o, en caso contrario (cuando las barras tienen idéntica dirección), la cercha es inestable. Esto permite calificar un nudo variable como bien conectado si a él acometen tres barras o más. Una definición análoga (pero ligeramente más complicada) se da para los nudos fijos. Gracias a ello, las soluciones candidatas se reducirán a las cerchas formadas exclusivamente por nudos bien conectados, lo que deja fuera algunas cerchas isostáticas (sin excluir por ello las óptimas) pero manteniendo dentro algunas que no lo son (a pesar de la "buena conexión" de sus nudos).

\subsection{Cambio de la posición de nudos}

El generador más inmediato es cambiar la posición de un nudo, tal y como el P de la Fig. 12. El nudo se elige al azar (del mismo modo en que se elegían ciudades) y, después, se eligen nuevas coordenadas dentro de márgenes previamente convenidos mediante series de números aleatorios con ecuaciones análogas a la (2). La nueva "configuración" obtenida se analiza, se evalúa su volumen estructural y se rechaza o acepta con el oráculo, ecuación (3), de manera idéntica a como se hizo para los itinerarios.
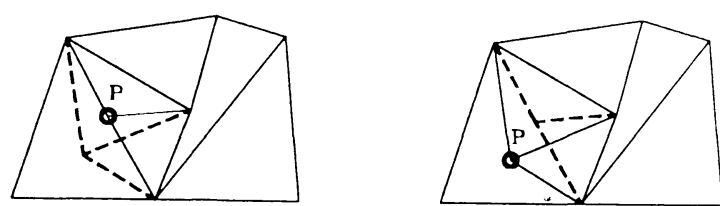

Fig.12.- Cambio de la posición de nudos.

\subsection{Cambio de barras}

En primer lugar una barra particular, tal como la $\mathrm{AB}$ a la izquierda de la Fig. 13, debe poder cambiar de posición. Como el número de barras es menor que el número total de posiciones posibles, siempre hay posiciones libres a donde una barra puede mudarse, tal como las dos señaladas a trazos. El problema es que la cercha resultante puede no ser isostática para una posición particular de las libres. Para conseguir una cercha isostática, no parece haber otra forma que probar (cuando sea necesario) todas las posibilidades.

A tal fin, para cada barra y posición libre elegidas al azar, se analiza la cercha, si es isostática se calcula su volumen y se acepta o se rechaza con el oráculo. Pero, en caso contrario, se realiza otra elección al azar entre las posibilidades restantes, hasta encontrar una nueva cercha isostática o ninguna, en cuyo caso la cercha queda inalterada.

El resultado es, obviamente, un generador parsimonioso, pero que en el peor de los casos, necesitará un número de operaciones finito y acotado por una potencia del número total de nudos, aunque el exponente puede llegar a ser seis.

\subsection{Aumento y disminución del número de nudos}

El generador restante debiera ocuparse de variar el número de nudos de la cercha entre los límites fijados por el problema. Para quitar o añadir un nudo, deben quitarse o añadirse también dos barras a fin de cumplir la condición necesaria para el isostatismo. Esta operación, por supuesto, se refiere en exclusiva a los nudos variables, que al estar bien conectados serán extremo de tres barras o más

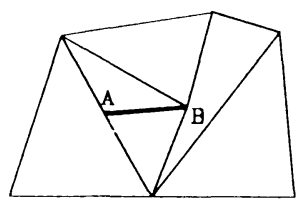

BARRA CANDIDATA

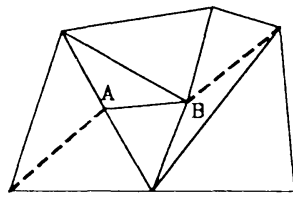

POSICIONES LIBRES

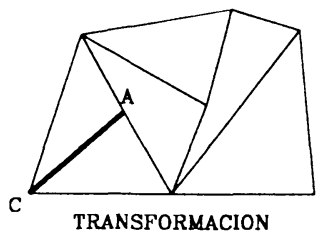

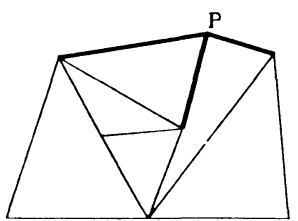

NUDO CANDIDATO CON TRES BARRAS

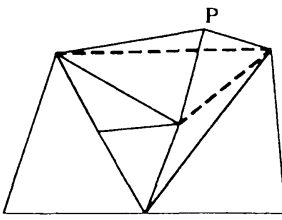

DOS POSICIONES PARA RESTITUIR UNA BARRA

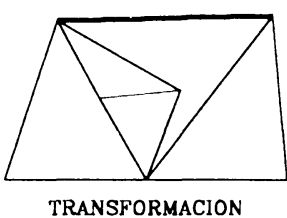

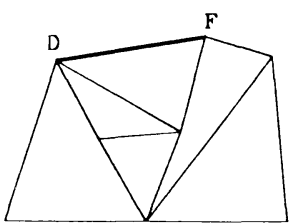

BARRA CANDIDATA

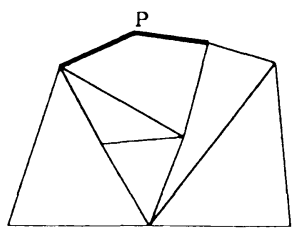

DIVISION EN DOS BARRAS

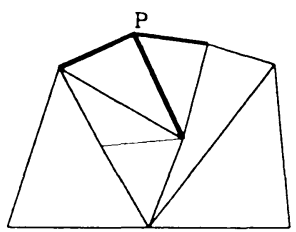

RESTITUCION DE EA BARRA

Fig.13.- Generadores que alteran la conexión de nudos por barras: De izquierda a derecha: cambio de posición dētina barra, supresión de un nudo; y adición de un nudo. 
Para suprimir un nudo puede elegirse al azar uno cualquiera con tres barras, tal y como el $\mathrm{P}$ en el centro de la Fig. 13, suprimir sus tres barras y restituir una de ellas en una nueva posición, elegida también al azar. En el caso peor, puede que haya que explorar todas las posibilidades hasta encontrar una alternativa isostática. La supresión de un nudo resulta, en consecuencia, tan parsimoniosa como el cambio de una barra.

El procedimiento para añadir un nudo es bastante similar. El objetivo se cifra ahora en que el nuevo nudo tenga tres barras. Para ello se elige una barra al azar, tal como la DF a la derecha de la Fig. 13, se divide en dos mediante el nuevo nudo $\mathrm{P}$ (elegido también al azar) y se une éste con otro elegido al azar entre los restantes. En total se habrá añadido un nudo y dos barras. Una vez más será necesario explorar al azar todas las posibilidades para la barra candidata hasta encontrar una nueva cercha isostática. En el caso peor, la transformación es igualmente parsimoniosa.

Con estas reglas para añadir o suprimir un nudo, se obtiene tan sólo un ligero cambio en su número total. Con esto, sin embargo, las transformaciones cumplen todavía con las condiciones básicas anteriormente enunciadas: en particular, con la exigencia de que cualquier configuración $y$, por tanto, cualquier valor de los permitidos para el número de nudos pueda ser alcanzado tras un número finito de transiciones.

\subsection{Algunos resultados}

La mezcla de estos cuatro generadores constituye lo único necesario para proceder al recocido. Cada nueva cercha isostática será aceptada o rechaźada según la regla de selección y el esquema general del algoritmo se hace cargo del resto.

De los tres problemas canónicos mostrados en la Fig. 14, los correspondientes a uno, dos y cuatro pesos entre dos apoyos, la solución teórica del primero fue descubierta por Michell, como se indicó, quien demostró que era necesariamente óptima mediante un famoso teorema que lleva su nombre. El recocido alcanza a determinar esta solución (véase la Fig. 15) y las soluciones suministradas para los otros dos problemas presentan un indudable buen aspecto, y aunque seguramente no son óptimas en sentido absoluto pueden reputarse de muy buenas (de hecho, su volumen estructural coincide o mejora las mejores estimaciones disponibles, véase Cervera,1993). Nótese que las soluciones originales de Michell son mecanismos (o, si se prefiere, estructuras funiculares), mientras que aquí aparecen diagonales sin esfuerzo para asegurar el isostatismo. Pero como el dimensionado es estricto no contribuyen (casi) al volumen estrutural. En el problema M4, aunque se aprecian detalles michellianos y las líneas generales del diseño, no se consigue igual definición; en lenguaje corriente: la forma general es "satisfactoria", pero abundan las imperfecciones locales. Esta situación es bien conocida en la producción de acero o en la "producción" de soluciones para el problema del viajante de comercio: el recocido simulado identifica con precisión suficiente la región del óptimo, pero no el propio óptimo.

El avance del recocido hacia la solución de Michell puede ilustrarse con una serie de soluciones candidatas separadas por una decena de ciclos y que, en consecuencia, corresponden a temperaturas progresivamente más bajas, (Fig. 16). Si se considera la serie completa, resulta notable que la "primera decisión" sea disminuir la esbeltez de la estructura, es decir, la proporción entre el alto y el ancho de la cercha, tal y como se ve en la segunda fila de la lámina (con temperaturas todavía altas). Después se ajusta el ángulo que forman las barras en los apoyos en la cercanía de un ángulo recto (en la tercera fila), más tarde se "decide" que la solución debe ser simétrica respecto al eje horizontal (cuarta fila), y finalmente se van afinando los detalles geométricos. Se trata, desde luego, de unaforma metafórica de hablar: el algoritmo no toma más decisión que aceptar o rechazar cada nueva configuración, y aun ésta es una decisión escrita en el programa por quién lo redactó. En cualquier caso, esta secuencia en la toma de "decisiones", de mayor a menor importancia, ha sido ya destacada por otros investigadores en relación al recocido de muy diversos problemas (Press et alii, 1988:346-351).

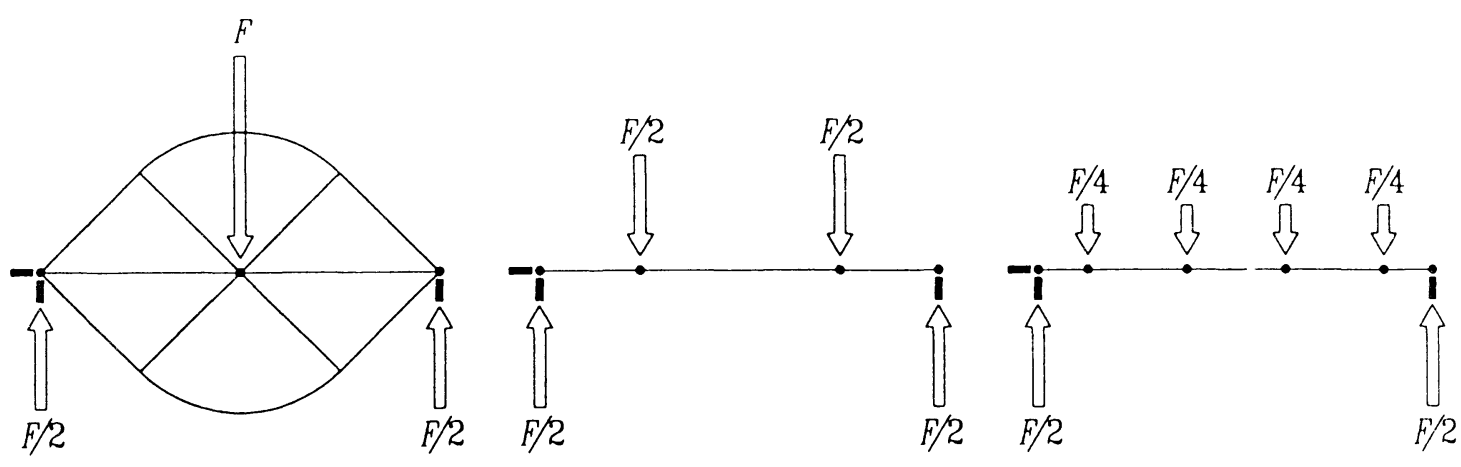

Fig.14.- Tres problemas de flexión simple: Se trata de problemas de Maxwell según la nomenclatura de Cērvera (1989), denominados, de izquierda a derecha, M1,M2 yM4. En el problema de la izquierda la solución óptima sigue el trasado generador indicado: para construirla hay quedibujar infinitos radios desde el punto de aplicación de la acción F hastalos dos arcos de círculo. 

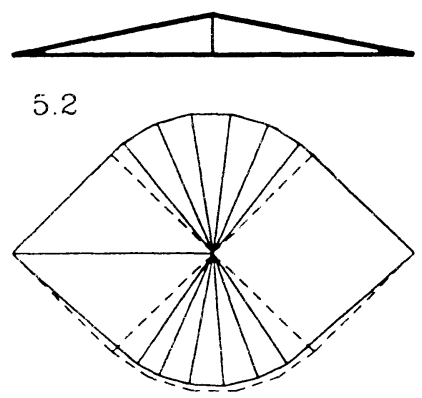

1.29126

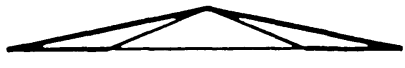

5.2

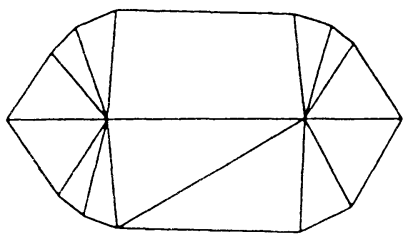

0.933375

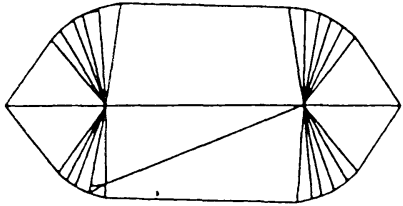

0.927545

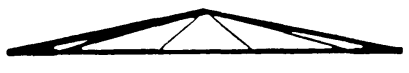

5.2

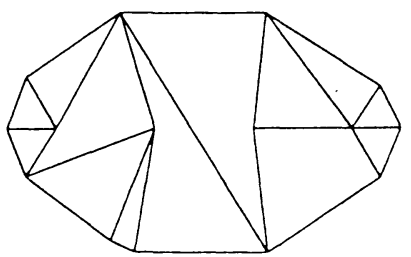

0.821029

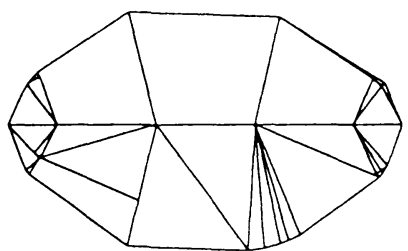

0.814416

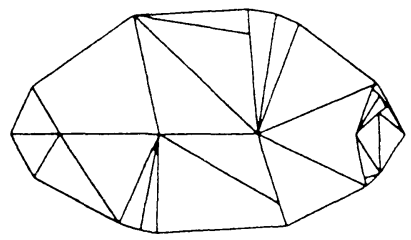

0.818566

Fig.15.- Soluciones estrictas en flexión simple. De arriba abajo: soluciones iniciales basadas en el "cuchillo español"; soluciones mejores de las series $M=15, M=30$ (sólo para dos y cuatro cargas) y $M=45$ (sólo para cuatro cargas). De izquierda a derecha: problemas M1, M2,y M4. En el caso de una carga se dibuja a trazos el trazado principal de la solución de Michell. El volumen estructural, que figura al lado de cada diseño, es adimensional, y debe multiplicarse por FL, siendo L la distancia entre apoyos. El espesor de las barras es proporcional al valor absoluto de la solicitación que soportan.

Hasta ahora las soluciones son teóricas, en la medida en que se considera a la tracción y a la compresión fenómenos equivalentes, $\mathrm{y}$ a que se admite que se podrá disponer en cada barra exactamente la mínima cantidad de material necesaria para cada forma. En la práctica, como se sabe, nada de esto es cierto: las barras comprimidas fallan con menor tensión que las traccionadas debido a fenómenos de inestabilidad, y para construir la cercha sólo se cuenta en general con catálogos limitados de perfiles. Cuando estos dos aspectos se tienen en cuenta, el recocido opera del mismo modo, mostrando formas que probablemente no son significativamente mejorables Fig. 17 y que se apartan, en ocasiones de manera notable, de las formas óptimas más teóricas Fig. 18.

Para $L=2 \mathrm{~m}$, la penalización por elemento mínimo tiene consecuencias evidentes: el número de barras es pequeño; en ocasiones, para conseguir convertir el mecanismo teórico en una cercha isostática es preferible una subestructura "rígida" a una diagonal (lo que ocurre en ambas soluciones para M2, diagonal $A B$ en la Fig. 17); la penalización es tanto peor cuanto más subdividida está la carga total (así M4 exige más volumen que M2, al revés que en el caso teórico); salvo en las soluciones a M1, toda referencia visual a las soluciones michellianas desaparece. Resulta notable el hecho de que, ahora, parece existir un mínimo absoluto para M4, al revés que en el caso teórico de la Fig. 15.Por el contrario, el espacio de M2 contiene al menos dos mínimos cuasióptimos. La penalización (entre pandeo y elemento mínimo) respecto al volumen estructural teórico se sitúa entre 1,34 y 2,13 .

En el caso $L=16 \mathrm{~m}$ (Fig. 18), la esbeltez aumentaría en ocho para cada barra si las soluciones aumentaran simplemente de tamaño; estodesplaza totalmente el óptimo, de manera que el aspecto visual no rememora el michelliano ni el de las soluciones pequeñas. La forma de los diseños puede explicarse sin dificultad: el cordón superior (comprimido) se subdivide por varios tirantes traccionados que distribuyen la carga a la vez que reducen su longitud 


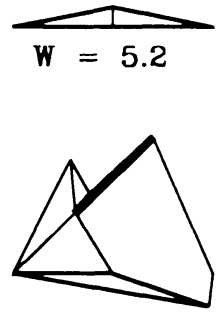

12.1958

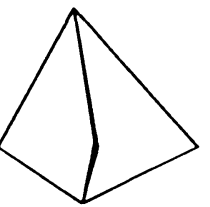

1.96669

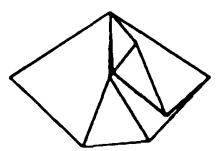

1.3985

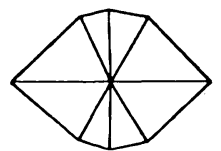

1.30803
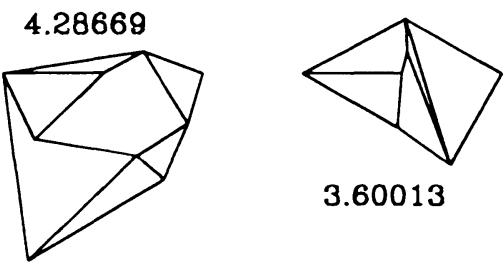

3.60013

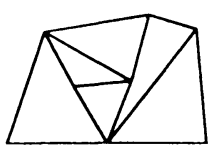

\subsection{7}
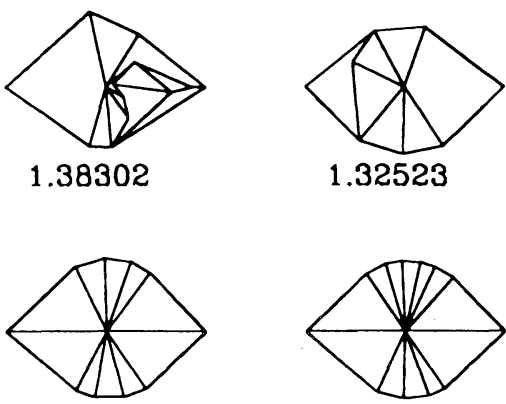

1.30047

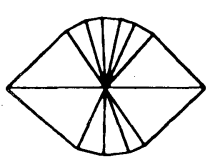

1.29592
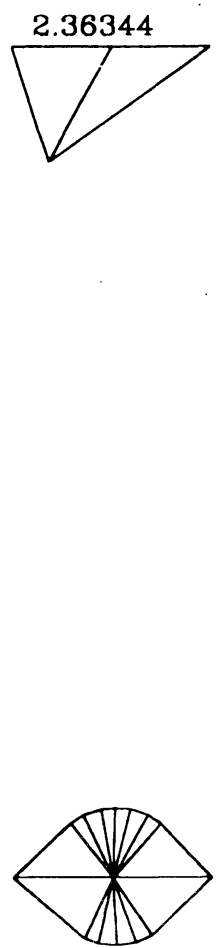

1.29365

Fig.16.- Avance del recocido hacia la solución de Michell.

de pandeo (M1 y M2). El cordón inferior (traccionado) puede ser tan largo como sea preciso.

En la solución M1, para asegurar el isostatismo de la cercha, se prefiere una subestructura "rígida" frente a la simple triangulación de alguno de los cuadriláteros inferiores. En la práctica corriente, este amasijo de pequeñas piezas se sustituirá por una pieza, tal y como un perfil IPE de los utilizados como vigas; de esta manera, la solución sugiere que el espacio de búsqueda quizás debe ampliarse a las estructuras hiperestáticas en las que los nudos se modelan como "rígidos" y nocomo articulaciones. Este es también el caso en la solución a M4, aunque aquí las dos formas (subestructura rígida o triangulación) están presentes de forma simultánea, una en la mitad izquierda y la otra en la derecha. Diseñando manualmente y procediendo por simetría, no se consiguió un diseño "homogéneo" que fuera mejor; la situación es, tal parece, como si ambas mitades se "ayudaran" mutuamente a fin de ajustarse mejor al catálogo. La penalización (entre pandeo y elemento mínimo) respectoal volumen estructural teórico se sitúa entre 2 y 3 .

Otra conclusión que resulta ahora obvia se refiere a la simetría. Desde que se abandona el universo teórico del dimensionado sin catálogo, las reglas del tipo "si las cargas son simétricas,las soluciones deben serlo" no son de aplicabilidad general. Esto se debe a la asimétrica barra que, en la solución de Michell, debe disponerse para asegurar el isostatismo. Puesto que el dimensionado ahora no es estricto, en esa barra (o en la parte de la cercha con idéntica función) hay que gastar un mínimo de material debido al elemento mínimo del catálogo. Si esa cantidad es importante, lo mejor es, al parecer, variar la forma para que al menos no se desperdicie inútilmente.

En todo caso resulta palpable que el volumen estructural no siempre captura las propiedades que se aprecian en los diseños viables (y realmente construidos) considerados como mejores ${ }^{2}$.

2. Nótese aquí que, al menos en nuestro país y sin entrar en mucho detalle, el volumen estructural es la medida usada en la práctiea corriente para los diseños en acero laminado; esto es debido a que el precio de tales estructuras setasa mediante un precio por unidad de masa, siendo ésta, denuevo, proporcional al peso, al volumen, o al volumen estructural. En consecuencia, en este caso, el precio resultará tan "ingenuo" como pueda serlo el volumen; y, al revés, cualquier corrección menor que se introduzca en aquél puede aplicarse a éste. 


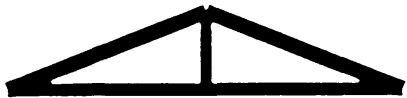

5967.56

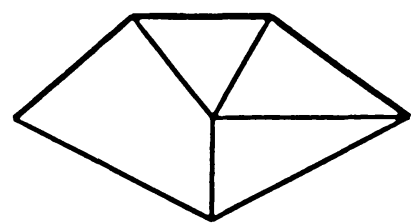

3441.82

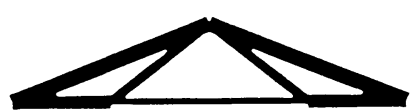

6023.87

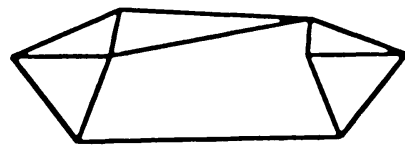

3354.39

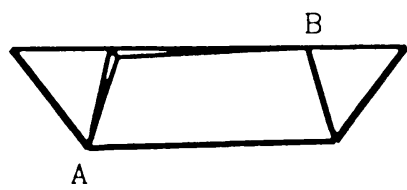

3348.51

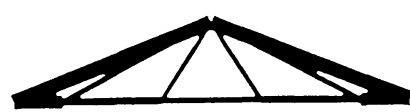

6146.8

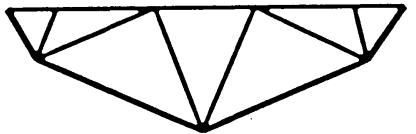

3478.78

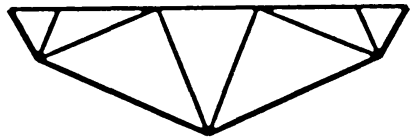

3476.65

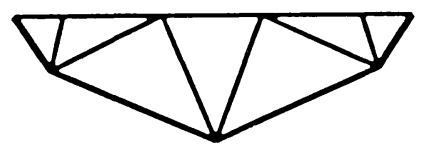

3479.62

Fig.17.- Soluciones para $L=2 m$ ytubo hueco: La ordenación de los diseños es la misma que en la figura 15 . El volumen estructural figura en $\mathrm{kN} \cdot \mathrm{mm}$. Las barras se dimensionan con tubos redondos huecos españoles y se comprueban según la norma $M V$, considerando un acero A42b. Para F se eligió el valor de $100 \mathrm{kN}$.

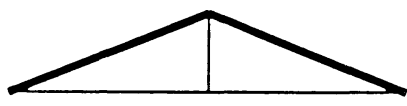

114580

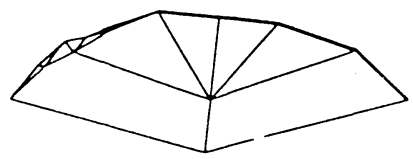

43556.1

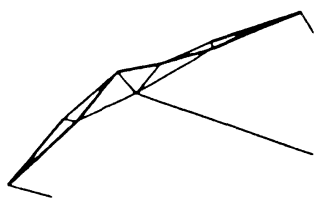

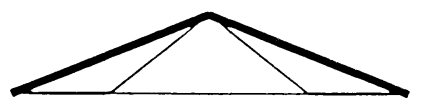

115030

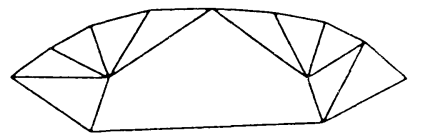

37460

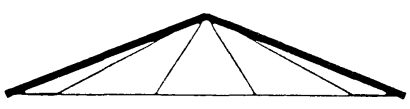

116014

37448.4
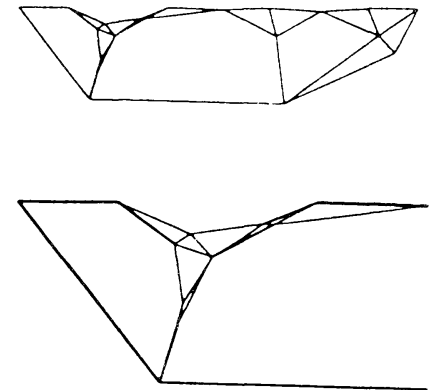

Fig.18.- Soluciones para $L=16$ mytubo hueco $:$ Las soluciones, aunque dibujadas al mismo tamaño en el papel, son "ocho"veces más grandes que las anteriores. Arriba: soluciones iniciales. Centro: soluciones mejores. Abajo: detalle a tamaño doble de las zonas que aseguran el isotatismo de las soluciones para M1 y M4. Los convenios son los mismos que en la figura anterior. 


\section{Conclusión}

Los resultados mostrados hasta aquí son sólo una pequeña parte de los obtenidos mediante recocido hasta la fecha (véanse más ejemplos en Vázquez, 1994). Los problemas abordados son muy distintos entre sí y constituyen un amplio abanico del tipo de problemas de la teoría de estructuras. Resulta notable que en todos ellos el mismo algoritmo (con la excepción de los generadores de cambios al azar y de lafunción objetivo) alcance a obtener soluciones cuasióptimas.

Cabe decir que, en general, los problemas estudiados, tal y comolaoptimación de cerchas, presentan una característica nueva para el algoritmo de recocido: la heterogeneidad de los parámetros que definen la función que se desea optimar. Tanto en el problema del viajante como en las simulaciones termodinámicas, los parámetros que definen una configuración son usualmente homogéneos aunque numerosos, y diseñada una transformación para uno de ellos, vale para todos los demás. En los problemas de la teoría de estructuras, por el contrario, distintas clases de parámetros intervienen en la definición de la energía o de la función objetivo pertinente. En consecuencia, hay que diseñar un generador específico para cada categoría de variables $y$, también, decidir la frecuencia relativa con que debe usarse cada generador particular. Lo cierto es que la heurística de cada problema ha sugerido en cada ocasión reglas de sentido común para ello, y el algoritmo de recocido ha funcionado esencialmente bien siempre. En conclusión, como reza el título de estas líneas, se cuenta desde ahora con un nuevo algoritmo para resolver aproximadamente problemas de optimación en la teoría de estructuras.

Antes de acabar, quisiera llamar la atención sobre dos conclusiones laterales, sugeridas por los resultados obtenidos mediante "el primer uso" del algoritmo, y muy relacionadas con su uso futuro.

Como comenté al principio, en problemas termodinámicos se usa el recocido simulado, y otras técnicas afines, para probar si las funciones matemáticas elegidas representan bien los fenómenos reales, mientras que cuestiones tales como cuál sea la configuración estable fundamental de un cierto sistema se estudian mejor mediante el método experimental.

En teoría de estructuras el acento suele ser el contrario: por ello, suponiendo que ciertas funciones matemáticas representan bien los objetivos perseguidos, el recocido se usó para alcanzar éstos. Sin embargo, los resultados muestran que algunas son funciones ingenuas, y las formas optimas obtenidas, aunque correctas desde un punto de vista matemático, no son las que esperaríamos encontrar o las que un diseñador experto proyectaría (Fig. 2). Aquí el problema no es tanto optimar una función sino, más bien, encontrar la función matemática que represente fielmente los objetivos del diseño, que en actividades como la ingeniería y la arquitectura no siempre pueden explicitarse, mucho menos darles forma algebraica.

Debido a la universalidad y simplicidad del algoritmo de recocido, cabe esperar que, mediante su uso, algunos investigadores en optimación de estructuras se animen a poner el acento sobre el diseño de funciones (y quizás en enjuiciar los objetivos perseguidos), equilibrandoun balance que actualmente está muy inclinado a favor de los métodos puramente matemáticos para optimarlas. Una vez pueda comprobarse mediante el algoritmo de recocido ( $u$ otros de iguales características) la adecuación de una función a un propósito concreto (y el merecimiento de este último), valdrá la pena, obviamente, considerar sus particulares características e investigar un algoritmo heurístico para su explotación práctica.

El segundo aspecto que me gustaría comentar tiene que ver con la relativa utilidad de los algoritmos. Lo importante aquí es subrayar como el análisis heurístico construye propiedades sintéticas de mucho interés, allí donde un algoritmo automático robusto (como el recocido) sólo muestra un resultado final desprovisto de propósito (Fig. 2, abajo a la derecha). En la serie que ilustra comoel recocido determina la solución de Michell (Fig. 16), reconocemos una cierta jerarquía de decisiones. Peroesto es gracias a que la teoría de diseño de estructuras-bien ilustradaen manuales como el de Cervera (1993) anteriormente citado- ha determinado previamente las propiedades sintéticas que debe exhibir la solución óptima. Con esta teoría hemos aprendido a "mirar". Un diseñador experimentado puede usar estas propiedades para obtener excelentes aproximaciones o, cuando otros objetivos así lo exijan, predecir cuánto se apartará su diseño del óptimo teórico. Y, por supuesto, sin necesitar poderosas máquinas para ello. Sin embargo, láminas semejantesa laFig. 16 para problemas nuevos o inexplorados quizá puedan sugerir a la teoría de diseño qué propiedades sintéticas deben investigarse y su importancia relativa. Pero será la teoría de diseño de estructuras quien facilitaráal diseñador las reglas heurísticas para un fértil y rápido proyecto. No un algoritmo.

\section{REFERENCIAS}

AROCA, RICARDO. (1994). "Conversación personal".

ATREK E. ET ALII: R. H. GALTAGHER, K. M. RAGSDELL Y O. C. ZIENKIEWICZ. (1984). "New Directions in Optimun Structural Design". Chichester: John Wiley et Sons.

CANDELA, FÉLIX. (1985). "En defensa del formalismo y otros escritos".s.1.: Xarait Ediciones. 
CERVERA, JAIME. (1989) "Tres teoremas fundamentales de la teoría del diseño de estructuras". Informes de la Construcción, v. $40, n^{0} 399$, pp. 57-66.

CER VERA, JAIME. (1993). "Diseño de estructuras en edificación". Madrid: Instituto Juan de Herrera \& Departamento de Estructuras de Edificación (ETSAM).

CROSS, HARDY. (1936) "The relation of analysis to structural design” Trans. Am. Soc. Civil Eng.n ${ }^{\circ}$ 62, pp. 1.3631.408.

GAREY, MICHAEL R. ET JOHNSON, DAVID S. (1979)

"Computers and Intractability". New York: Freeman.

HAJEK, B. (1988). "Cooling Schedules for Optimal Annealing" Math. Oper. Res. vol. 13, pp. 311-329.

HANSON, DIRK. (1982). "The New Alchemists". s.d., versión castellana de Paloma Villegas, "Los nuevos alquimistas' Silicon Valley y la revolución microelectrónica, Barcelona: Planeta, (1984).

KIRKPATRICK, SCOTT ET ALII: C.D. GELATT Y MARIO P VECCHI. (1983). "Optimization by Simulated Annealing" Science, vol. $220, \mathrm{n}^{\circ} 4.598$, pp. 671-680.

LIN, S. ET B. W. KERNIGHAN. (1973). “An Effective Heuristic Algorithm for the Traveling-Salesman Problem", Operations Research, $n^{\circ} 21$, pp 498-516.

METROPOLIS, NICHOLAS ET ALII: ARIANNA W. Y MARSHALL N. ROSENBLUTH; Y AUGUSTA H. TELLER.
(1953). "Equation of State Calculations by Fast Computing Machines", The Journal of Chemical Physics, vol. 21, $\mathrm{n}^{\circ} 6$, pp. 1.087-1.092.

MICHELL, A.G.M. (1904). "The limits of Economy of Material in Frame-structures", Philosophical Magazine, S. 6, vol. 8, $\mathrm{n}^{\circ}$ 47.

MIGUEL, JOSE LUIS DE. (1974). "Trabajo estructural. Un nuevo escalar de las estructuras", Madrid: Escuela Téenica Superior de Arquitectura, tesis doctoral.

PRESS, WILLIAM H. ET ALII: BRIAN P. FLANNERY, SAUL A. TEUKOLSKY, Y WILLIAM T. VETTERLING. (1988). "Numerical Recipes in C. The Art of Scientific Computing", Cambridge: Cambridge University Press.

QUINTAS, VALENTÍN. (1992). "Sobre las formas de mínimo volumen de las celosías de sección constante", Informes de la construcción, vol. $43, n^{\circ} 418$, pp. $61-77$.

SCHRÖDINGER, ERWIN. (1944). "What is life?"se cita la reimpresión conjunta con Mind and Matter", London: Cambridge University Press, 1967

VANDERPLAATS, G.N. (1984). "Numerical Methods for Shape Optimization: An Assessment of the State of the Art" en ARTEK et alii, 1984:89-103.

VÁZQUEZ, MARIANO. (1994). "Recocido simulado: un nuevo algoritmo para la optimación de estructuras", Madrid: Universidad Politécnica, tesis doctoral, AA 94/95.

\section{Publicación del Instituto Eduardo Torroja-CSIC}

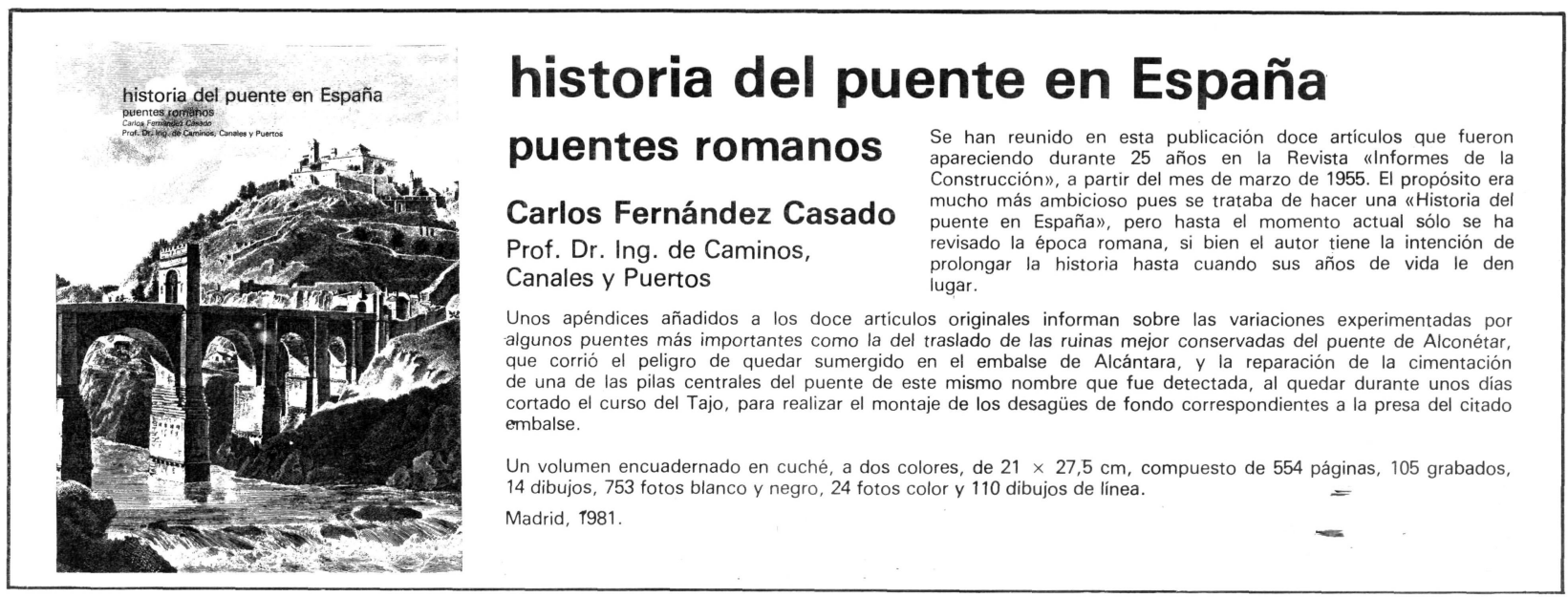

